\title{
Approximation Properties of a Certain Modification of Durrmeyer Operators
}

\author{
Asha Ram Gairola \\ Doon University \\ Karunesh Kumar Singh \\ Institute of Engineering and Technology, Lucknow \\ Hassan Khosravian Arab \\ University of Isfahan \\ Vishnu Narayan Mishra ( $\nabla$ vishnunarayanmishra@gmail.com ) \\ Indira Gandhi National Tribal University, Lalpur, Amarkantak 484 887, M.P., India \\ https://orcid.org/0000-0002-2159-7710
}

\section{Research Article}

Keywords: Bernstein-Durrmeyer operator, integrable functions

Posted Date: May 18th, 2021

DOI: https://doi.org/10.21203/rs.3.rs-522598/v1

License: (1) (1) This work is licensed under a Creative Commons Attribution 4.0 International License. Read Full License 


\section{APPROXIMATION PROPERTIES OF A CERTAIN MODIFICATION OF DURRMEYER OPERATORS}

Asha Ram Gairola ${ }^{1}$, Karunesh Kumar Singh ${ }^{2}$, Hassan Khosravian-Arab ${ }^{3}$, Vishnu Narayan Mishra*

${ }^{1}$ Department of Mathematics, Doon University, Dehradun-248001

(Uttarakhand), India, Email: ashagairola@gmail.com

${ }^{2}$ Department of Applied Sciences and Humanities

Institute of Engineering and Technology

Lucknow-226021(Uttar Pradesh), India, Email: kksiitr.singh@gmail.com

${ }^{3}$ Department of Applied Mathematics and Computer Science, Faculty of Mathematics and Statistics, University

of Isfahan, 81746-73441 Isfahan, Iran.

Email: h.khosravian@sci.ui.ac.ir

*Department of Mathematics, Indira Gandhi National Tribal University, Amarkantak, Anuppur 484 887, Madhya

Pradesh, India, Email:vishnunarayanmishra@gmail.com

Abstract. We study approximation properties of a new operator $D_{n}^{M, 1}(f, x)$, introduced by Acu et al. in [Results Math 74:90, (2019)] for Lebesgue integrable functions in $[0,1]$. An error estimate by the Bézier variant of the operators $D_{n}^{M, 1}(f, x)$ is also obtained for the functions of bounded variation. By relevant numerical examples, the orders of approximation by the operator $D_{n}^{M, 1}(f, x)$ and the modified-Bernstein-Durrmeyer operator are also compared.

\section{INTRODUCTION}

The famous Weierstrass's theorem states that "A continuous function $f(x)$ in a compact domain $[a, b]$ can be uniformly approximated by polynomials". There are several proofs of this theorem available and we refer the reader to(see [24]). The main difficulty in the most of the proofs is that they are implicit and lack practical applications. On the other hand, Bernstein in 7 gave a constructive proof of the Weierstrass's theorem by introducing the classical Bernstein polynomials

$$
B_{n}(f)(x):=\sum_{k=0}^{n} f\left(\frac{k}{n}\right) p_{n, k}(x), \quad x \in[0,1]
$$

which are defined for a bounded function $f$ on $[0,1]$. Here the Bernstein basis

$$
p_{n, k}(x):= \begin{cases}\left(\begin{array}{l}
n \\
k
\end{array}\right) x^{k}(1-x)^{n-k}, & 0 \leq k \leq n, \\
0, & k>n, \text { or } k<0 .\end{cases}
$$

It is known that (see 8, 24, 25]) at a point $x$ of continuity of $f$ there holds the $\operatorname{limit} \lim _{n \rightarrow \infty} B_{n}(f)(x)=f(x)$. These operators have been widely studied by researchers $([1],[3], 5], ~ 9], ~ 29])$. Interesting properties of these operators include, shape preservation, uniform approximation, numerical quadrature, possibility of approximation of derivatives $f^{(s)}$ of a differentiable function $f$ by the corresponding derivative $\left(B_{n}(f)\right)^{(s)}$ etc. For the detailed results about the polynomials, $B_{n}(f)(x)$ it is worth refer to [8] and [25. The classical Bernstein polynomials $B_{n}(f)(x)$ have been a model for the study of various problems of approximation theory. In fact, many of the problems have been studied for Bernstein polynomials initially and later on extended to other linear positive operators.

The symbol $\mathbb{N}$ represents set of positive integers. Let $C[0,1]$ denote the normed space of continuous function defined on $[0,1]$ wherein the defining norm is given by $\|f\|:=\sup _{0<x<1}|f(x)|$. Here and subsequently, $L_{B}[0,1]$ denotes the class of Lebesgue integrable and bounded functions in $[0,1]$. The symbol $L(f)(x)$ or $L(f ; x)$ will denote the value of $L f$ at $x$ i.e. $L f(x)$.

${ }^{*}$ Corresponding author

Keywords: Rate of convergence, Approximation by positive operators, Approximate quadratures, Bounded variation, Modulus of continuity.

2010 AMS Subject classification:41A25, 41A36, 41A55, 41A10 . 
Definition 1. We write $f(x)=o\left(\frac{1}{n}\right)$ whenever there holds the limit $n f(x) \rightarrow 0$ as $n \rightarrow \infty$. Similarly, $f(x)=O\left(\frac{1}{n}\right)$ implies that $n f(x) \rightarrow g(x)$ as $n \rightarrow \infty$, where $g(x) \neq 0$.

Definition 2. The notation $f(x) \sim g(x)$ implies that there exist positive constants $A$ and $B$ such that $f(x) \leqslant B g(x)$ and $g(x) \leqslant A f(x)$. The constants $A, B$ being independent of $x$.

Definition 3. [24] For a function $f(x)$ continuous in the interval $[a, b]$, the modulus of continuity is defined as

$$
\omega(f, \delta):=\max _{|x-y| \leqslant \delta}|f(x)-f(y)|, \quad a \leqslant x, y \leqslant b .
$$

\section{Literature Review}

Although the polynomials $B_{n}(f)(x)$ are easy to work with, they lack rapid convergence. The highest rate of convergence is $O\left(n^{-1}\right)$ which is obtained for the linear functions only. In the case of approximation by Bernstein operators, we have the following upper bound for the rate of convergence.

Theorem 1. [25]For each $f \in C[0,1]$

$$
\left\|f-B_{n} f\right\| \leq \frac{5}{4} \omega\left(f, \frac{1}{\sqrt{n}}\right)
$$

In an attempt to improve the order of approximation, P. L. Butzer [10 suggested the process of linear combinations of Bernstein operators. For Bernstein polynomials these combinations $B_{n}(f, k,$.$) are defined by$

$$
B_{n}(f, k, x):=\sum_{j=0}^{k} C(j, k) B_{d_{j} n}(f, x),
$$

where

$$
C(j, k)=\prod_{i=0, i \neq j}^{k} \frac{d_{j}}{d_{j}-d_{i}}, k \neq 0 \text { and } C(0,0)=1,
$$

$d_{0}, d_{1}, \ldots d_{k}$ being $(k+1)$ arbitrary but fixed distinct positive integers. Although there is significant improvement in degree of approximation by the method of linear combinations, it requires values of $f$ at additional nodes $k / d_{j} n, k=\overline{0, n}, j=\overline{0, k}$. This requirement makes this method impractical for actual implementation. On the other hand the method of iterative combinations overcome this drawback. This method for improving the order of approximation have been suggested by Micchelli 27] which consider the combinations, $I-\left(I-B_{n}\right)^{k}$ of iterates of Bernstein polynomials. This combination can be viewed as a genuine iterations process. Agrawal and Gupta 6 applied the technique of iterative combinations to improve the order of approximation of Phillips operators. It is worth to mention recent paper 13 of Draganov wherein the author has established upper estimates in weighted simultaneous approximation by iterative combinations of the Bernstein polynomials. It is worth pointing out that Draganov's results includes rate of convergence in ordinary approximation as a particular case.

In this direction, recently, Arab et al. 23] have given yet another approach to enhance the rate of convergence by the Bernstein polynomials. Their method suggests splitting of the basis function $p_{n, k}(x)$ in following manner. We have the identity

$$
p_{n, k}(x)=(1-x) p_{n-1, k}(x)+x p_{n-1, k-1}(x) .
$$

We replace the function $(1-x)$ by $a(x, n)$ where $a(x, n)=a_{0}(n)+a_{1}(n) x$ and $a_{0}(n), a_{1}(n)$ are unknown sequences to be determined by suitable conditions. A new basis function $p_{n, k}^{M, 1}(x)$ is defined by

$$
p_{n, k}^{M, 1}(x):=a(x, n) p_{n-1, k}(x)+a(1-x, n) p_{n-1, k-1}(x) .
$$

Finally, a new Bernstein type operator $B_{n}^{M, 1}(f ; x)$ is defined by

$$
B_{n}^{M, 1}(f ; x):=\sum_{k=0}^{n} p_{n, k}^{M, 1}(x) f\left(\frac{k}{n}\right) .
$$

Arab et al. 23] proved the convergence of $B_{n}^{M, 1}(f ; x)$ to $f(x)$ in case the sequences $a_{i}(n), i=0,1$ satisfy certain conditions.

The Bernstein polynomials $B_{n}(f)(x)$ are not suitable for approximation of a larger class of functions, namely class of the Lebesgue integrable functions. Therefore, the operators $B_{n}(f)(x)$ are suitably modified for this purpose. The 
first such modification was introduced by L. V. Kantorovich(see 22, 31]). These so called Kantorovich operators and defined by

$$
K_{n}(f)(x):=(n+1) \sum_{k=0}^{n} p_{n, k}(x) \int_{\frac{k}{n+1}}^{\frac{k+1}{n+1}} f(t) \mathrm{d} t .
$$

Another modification of Bernstein operators (1.1) have been independently introduced by Durrmeyer 14 and Lupaş 26 by

$$
D_{n}(f ; x):=\int_{0}^{1}\left((n+1) \sum_{k=0}^{n} p_{n, k}(x) p_{n, k}(t)\right) f(t) \mathrm{d} t .
$$

The operators $D_{n}(f ; x)$ are obtained by replacing the values $f(k / n)$ by the weighted mean values

$$
(n+1) \int_{0}^{1} p_{n, k}(t) f(t) \mathrm{d} t
$$

of $f$ on $[0,1]$. We would like to mention that the notation $M_{n}(f, x)$ is also common for the operators (2.1). For a continuous function $f$ on $[0,1]$, it was proved by Derrienic that an upper estimate

$$
\sup _{x \in[0,1]}\left|M_{n} f(x)-f(x)\right| \leq 2 \omega_{f}(1 / \sqrt{n}), \quad n \geq 3
$$

holds.

The operators $D_{n}(f ; x)$ have been studied by Derriennic [15]- 16] and many other researchers(see [4], [?], [17]). In fact the operators (2.1) are obtained by setting $c=-1$ in the formula

$$
M_{n}^{c}(f, x)=(n-c) \sum_{k=0}^{\infty} p_{n, k}^{c}(x) \int_{0}^{\infty} p_{n, k}^{c}(t) f(t) \mathrm{d} t
$$

where

$$
p_{n, k}^{c}(x)=(-1)^{k} \frac{x^{k}}{k !} \phi_{n, c}^{(k)}(x) .
$$

The functions $\phi_{n, c}^{(k)}(x)$ are defined by

$$
\phi_{n, c}^{(k)}(x)= \begin{cases}(1-x)^{n}, & c=-1, \\ e^{-n x}, & c=0, \\ (1+x)^{-n}, & c=1 .\end{cases}
$$

This interesting and significant formula 2.2 was introduced by M. Heilmann in her thesis (see [21]) and its importance lies in the fact that the formula $(2.2)$ yields many important operators of approximation theory for different values of $c$.

It is interesting to observe that the authors of 18 has established following representation

$$
D_{n}(f, x):=(n+1) \int_{0}^{1} f(t)[(1-x)(1-t)]_{2}^{n} F_{1}\left(-n,-n ; 1 ; \frac{x t}{(1-x)(1-t)}\right) \mathrm{d} t .
$$

of the operators $D_{n}(f ; x)$ in terms of the hypergeometric function. This representation enables us to compute the images of monomials $e^{i}=t^{i}, i \in \mathbb{N} \cup\{0\}$ by the formula

$$
D_{n}\left(e_{i}, x\right)=\frac{\Gamma(n+2) \Gamma(i+1)}{\Gamma(n+i+2)}{ }_{2} F_{1}(-n,-i ; 1 ; x), \quad n>0, i>-1 .
$$

Another interesting integral variants of Bernstein operator have been studied in [19] and 20].

It turns out that the sequence $D_{n}(f, x)$ converges point wise to $f(x)$ at each point $x$ where $f$ is continuous. In fact(see [16]) for continuous function $f$ in $[0,1]$ and $n \geqslant 3$ there holds the estimate

$$
\sup _{x \in[0,1]}\left|D_{n} f(x)-f(x)\right| \leqslant 2 \omega\left(f, n^{-1 / 2}\right) .
$$

For a continuous functions $f$ on $[0,1]$, the convergence of $D_{n}(f, x)$ to $f(x)$ is uniform. For twice differentiable function $f(x)$, we have following theorem:

Theorem 2. [16] If $f$ is integrable, bounded on $[0,1]$ and admits a second derivative at point $x \in[0,1]$, then

$$
\lim _{n \rightarrow \infty} n\left(M_{n} f(x)-f(x)\right)=(1-2 x) f^{\prime}(x)+x(1-x) f^{\prime \prime}(x) .
$$

Moreover, this limit is uniform if $f^{\prime \prime}$ is continuous over $[0,1]$. 
Therefore, the order of approximation by the operators $D_{n}(f, x)$ is saturated by $O\left(n^{-1}\right)$. It is of interest to know that the operators $D_{n}(f, x)$ are even suitable for $p$-integrable functions i.e. if $f \in L_{p}[0,1]$ then $D_{n}(f, x)$ are approximation process and the limit $D_{n}(f, x) \rightarrow f(x)$ holds $([12)$. We mention some important properties of the operators $D_{n}(f, x)$.

(1) $D_{n}(f, x)$ is linear and positive;

(2) $D_{n}(f, x)$ is convex for a convex function $f(x)$;

(3) for differentiable function $f$ there holds

$$
\lim _{n \rightarrow \infty} \frac{d^{s}}{d x^{s}}\left(D_{n}(f, x)\right)=f^{(s)}(x)
$$

(4) it preserves the constants;

(5) $D_{n}(f, x)$ preserves the degree of polynomials when their degrees are $\leq n$;

(6) it is a contraction on $L_{p}[0,1], p \geq 1$.

In this paper, we obtain rate of approximation for a new integral variant $D_{n}^{M, 1}(f, x)$ of the Bernstein polynomial $B_{n}(f)(x)$. We will also compare the degrees of approximation by the operators $D_{n}^{M, 1}(f, x)$ and $D_{n}(f, x)$ for appropriate functions.

\section{Construction of Operator}

Let $f \in L_{B}[0,1]$. Using the new Bernstein type basis functions $p_{n, k}^{M, 1}(x)$ defined in 23 the operator $D_{n}^{M, 1}(f, x)$ is defined by

$$
D_{n}^{M, 1}(f, x):=\frac{n(n+1)}{\left(2 a_{0}(n)+a_{1}(n)\right) n+2\left(a_{0}(n)+a_{1}(n)\right)} \sum_{k=0}^{n} p_{n, k}(x) \int_{0}^{1} p_{n, k}^{M, 1}(t) f(t) \mathrm{d} t
$$

where

$$
p_{n, k}^{M, 1}(t)=a(t, n) p_{n-1, k}(t)+a(1-t, n) p_{n-1, k-1}(t) .
$$

The function

$$
a(t, n)=a_{1}(n) t+a_{0}(n)
$$

and the unknown sequences $a_{0}(n), a_{1}(n)$ are to be determined. Here the monomial $e_{i}(t)=t^{i}$, for each $i=0,1,2$. For simplicity of notation, we write $N\left(a_{0}(n), a_{1}(n)\right)$ instead of $\frac{n(n+1)}{\left(2 a_{0}(n)+a_{1}(n)\right) n+2\left(a_{0}(n)+a_{1}(n)\right)}$. Write

$$
D_{n}^{M, 1}(f, x)=A_{n}(f, x)+B_{n}(f, x),
$$

where

$$
A_{n}(f, x)=N\left(a_{0}(n), a_{1}(n)\right) \sum_{k=0}^{n} p_{n, k}(x) \int_{0}^{1} a(t, n) p_{n-1, k}(t) f(t) \mathrm{d} t
$$

and

$$
B_{n}(f, x)=N\left(a_{0}(n), a_{1}(n)\right) \sum_{k=0}^{n} p_{n, k}(x) \int_{0}^{1} a(1-t, n) p_{n-1, k-1}(t) f(t) \mathrm{d} t .
$$

We will make frequent use of following identities

$$
\sum_{k=0}^{n} k^{\underline{N}} p_{n, k}(x)=n^{\underline{N}} x^{N}, \quad N \leq n
$$

and

$$
\int_{0}^{1} t^{m} p_{n, k}(t) \mathrm{d} t=\frac{n !(m+k) !}{k !(m+n+1) !}, \quad k, n, m \in \mathbb{N} .
$$

Here $k^{\underline{N}}$ denote the permutation $k(k-1) \cdots(k-N+1)$ for $k \geqslant N$. 


\section{Preliminaries}

We begin with the computation of moments of operator $D_{n}^{M, 1}(f, x)$.

Lemma 1. Let $e_{i}(t)=t^{i}, i=\overline{0,2}$. Then

$$
\begin{gathered}
D_{n}^{M, 1}\left(e_{0}, x\right)=1, \\
D_{n}^{M, 1}\left(e_{1}, x\right)=\frac{x\left(2 a_{0}(n)(n+2) n+a_{1}(n) n^{2}+4 a_{1}(n) n\right)+a_{0}(n)(n+2)+2 a_{1}(n)}{(n+2)\left(2 a_{0}(n)(n+1)+a_{1}(n)(n+2)\right)},
\end{gathered}
$$

and

$$
\begin{aligned}
D_{n}^{M, 1}\left(e_{2}, x\right) & =\frac{2 a_{0}(n)(n+3)+6 a_{1}(n)+\left(6 a_{0}(n) n(n+3)+2 a_{1}(n) n(n+9)\right) x}{(2+n)(3+n)\left(2 a_{0}(n)(1+n)+a_{1}(n)(2+n)\right)} \\
& +\frac{\left(2 a_{0}(n)(n+3)+a_{1}(n)(n+6)\right) n(n-1) x^{2}}{(2+n)(3+n)\left(2 a_{0}(n)(1+n)+a_{1}(n)(2+n)\right)} .
\end{aligned}
$$

Proof. The proof follows by straight forward calculations and applications of (3.2) together with (3.3).

Remark 1. If we make the assumptions $D_{n}^{M, 1}\left(e_{1}, x\right)=x$, then, we obtain $(n+2) a_{0}(n)+2 a_{1}(n)=0$. It is easily seen that Hence, we have

which implies

$$
D_{n}^{M, 1}\left(e_{2}, x\right)=\frac{n^{3} x^{2}+n^{2} x(3 x+2)+2 n x(5-2 x)+2}{n(n+2)(n+3)}
$$

$$
\begin{aligned}
D_{n}^{M, 1}\left(e_{2}, x\right) & =x^{2}+\frac{2+2\left(5 n+n^{2}\right) x+2\left(-5 n-n^{2}\right) x^{2}}{n(n+2)(n+3)} \\
& =x^{2}+\frac{2\left(1+n(n+5) \varphi^{2}(x)\right)}{n(n+2)(n+3)}
\end{aligned}
$$

Therefore,

$$
\begin{aligned}
D_{n}^{M, 1}\left(\left(e_{1}-x\right)^{2}, x\right) & =\frac{2\left(1+n(n+5) \varphi^{2}(x)\right)}{n(n+2)(n+3)} \\
& \leq \frac{C}{n+2} \varphi^{2}(x)
\end{aligned}
$$

where $C$ is a positive constant.

\section{Rate of Approximation}

Theorem 3. Let $D_{n}^{M, 1}(f, x)$ be the operator defined by 3.1) and $f \in C[0,1]$. If $a_{0}(n)$ and $a_{1}(n)$ are convergent sequences such that $(n+2) a_{0}(n)+2 a_{1}(n)=0$ then for any positive real number $m$,

$$
\begin{aligned}
& \left|D_{n}^{M, 1}(f, x)-f(x)\right| \\
& \leqslant \omega\left(f, \frac{1}{m}\right)\left|N\left(a_{0}(n), a_{1}(n)\right)\right|\left(\frac{m}{\sqrt{n}}\left(\left(\frac{1-3 \varphi^{2}(x)}{3}+\frac{x^{2}}{n}\right)^{1 / 2}+\left(\frac{1-3 \varphi^{2}(x)}{3}+\frac{(1-x)^{2}}{n}\right)^{1 / 2}\right)+\frac{2}{n}\right)
\end{aligned}
$$

Proof. Since, $D_{n}^{M, 1}\left(e_{0}, x\right)=1$, we have

$$
D_{n}^{M, 1}(f, x)-f(x)=A_{n}(f, x)+B_{n}(f, x) .
$$

We have that

$$
\begin{aligned}
\left|A_{n}(f, x)\right| & =\left|N\left(a_{0}(n), a_{1}(n)\right)\right|\left|a_{0}(n)+a_{1}(n)\right|\left|\sum_{k=0}^{n} p_{n, k}(x) \int_{0}^{1} p_{n-1, k}(t)(f(t)-f(x)) \mathrm{d} t\right| \\
& \leqslant\left|N\left(a_{0}(n), a_{1}(n)\right)\right|\left|a_{0}(n)+a_{1}(n)\right| \sum_{k=0}^{n} p_{n, k}(x) \int_{0}^{1} p_{n-1, k}(t) \omega(f,|t-x|) \mathrm{d} t \\
& \leqslant \omega\left(f, \frac{1}{m}\right)\left|N\left(a_{0}(n), a_{1}(n)\right)\right| \sum_{k=0}^{n} p_{n, k}(x) \int_{0}^{1} p_{n-1, k}(t)(m|t-x|+1) \mathrm{d} t .
\end{aligned}
$$


Here we have used the inequality(see [24])

$$
\omega(f, \lambda \delta) \leq(\lambda+1) \omega(f, \delta)
$$

which holds for any real positive number $\lambda$. An application of Hölder's inequalities for integration and then summation yields

$$
\begin{aligned}
\left|A_{n}(f, x)\right| & \leqslant \omega\left(f, \frac{1}{m}\right)\left|N\left(a_{0}(n), a_{1}(n)\right)\right|\left|a_{0}(n)+a_{1}(n)\right| \sum_{k=0}^{n} p_{n, k}(x)\left(m\left(\int_{0}^{1} p_{n-1, k}(t)(t-x)^{2} \mathrm{~d} t\right)^{1 / 2}\right. \\
& \left.\left(\int_{0}^{1} p_{n-1, k}(t) \mathrm{d} t\right)^{1 / 2}+\int_{0}^{1} p_{n-1, k}(t) \mathrm{d} t\right) \\
& =\omega\left(f, \frac{1}{m}\right)\left|N\left(a_{0}(n), a_{1}(n)\right)\right|\left|a_{0}(n)+a_{1}(n)\right|\left(m\left(\sum_{k=0}^{n} p_{n, k}(x) \int_{0}^{1} p_{n-1, k}(t)(t-x)^{2} \mathrm{~d} t\right)^{1 / 2}\right. \\
& \left.+\sum_{k=0}^{n} p_{n, k}(x) \int_{0}^{1} p_{n-1, k}(t) \mathrm{d} t\right) \\
& =\omega\left(f, \frac{1}{m}\right)\left|N\left(a_{0}(n), a_{1}(n)\right)\right|\left|a_{0}(n)+a_{1}(n)\right|\left(m\left(\frac{1-3 \varphi^{2}(x)}{3}+\frac{(1-x)^{2}}{n}\right)^{1 / 2} \frac{1}{\sqrt{n}}+\frac{1}{n}\right) .
\end{aligned}
$$

Proceeding likewise for $B_{n}(f, x)$, we get

$$
\left|B_{n}(f, x)\right| \leqslant \omega\left(f, \frac{1}{m}\right)\left|N\left(a_{0}(n), a_{1}(n)\right)\right|\left|a_{0}(n)+a_{1}(n)\right|\left(m\left(\frac{1-3 \varphi^{2}(x)}{3}+\frac{x^{2}}{n}\right)^{1 / 2} \frac{1}{\sqrt{n}}+\frac{1}{n}\right) .
$$

Combining the estimates 5.1 and 5.2 we get

$$
\begin{aligned}
& \left|D_{n}^{M, 1}(f, x)-f(x)\right| \\
& \leqslant \omega\left(f, \frac{1}{m}\right)\left|N\left(a_{0}(n), a_{1}(n)\right)\right|\left|a_{0}(n)+a_{1}(n)\right|\left(\frac{m}{\sqrt{n}}\left(\left(\frac{1-3 \varphi^{2}(x)}{3}+\frac{x^{2}}{n}\right)^{1 / 2}+\left(\frac{1-3 \varphi^{2}(x)}{3}+\frac{(1-x)^{2}}{n}\right)^{1 / 2}\right)+\frac{2}{n}\right)
\end{aligned}
$$

This completes the proof.

\section{Corollary 1. Let}

$$
\delta_{n}(x)=\max \left\{\frac{1-3 \varphi^{2}(x)}{3}+\frac{(1-x)^{2}}{n}, \frac{1-3 \varphi^{2}(x)}{3}+\frac{x^{2}}{n}\right\}
$$

then, by straight forward calculations

$$
\delta_{n}(x)= \begin{cases}\frac{1-3 \varphi^{2}(x)}{3}+\frac{(1-x)^{2}}{n}, & 0 \leqslant x \leqslant 1 / 2 \\ \frac{1-3 \varphi^{2}(x)}{3}+\frac{x^{2}}{n}, & 1 / 2 \leqslant x \leqslant 1\end{cases}
$$

Now, setting $m=\frac{1}{2 \sqrt{n \delta_{n}(x)}}$, we get

$$
\left|D_{n}^{M, 1}(f, x)-f(x)\right| \leqslant 6\left|N\left(a_{0}(n), a_{1}(n)\right)\right|\left|a_{0}(n)+a_{1}(n)\right| \omega\left(f, \sqrt{\frac{\delta_{n}(x)}{n}}\right) .
$$

Corollary 2. A particular solution of the equation $(n+2) a_{0}(n)+2 a_{1}(n)=0$ is

$$
a_{0}(n)=-\frac{2}{n}, a_{1}(n)=1+\frac{2}{n} .
$$

Using these values of $a_{0}(n)$ and $a_{1}(n)$ we have

$$
\left|D_{n}^{M, 1}(f, x)-f(x)\right| \leqslant 6\left(1+n^{-1}\right) \omega\left(f, \sqrt{\frac{\delta_{n}(x)}{n}}\right) .
$$

Corollary 3. If we suppose $(n+2) a_{0}(n)+2 a_{1}(n)=0$, then $N\left(a_{0}(n), a_{1}(n)\right)=\frac{(n+1)(n+2)}{a_{1}(n) n}$ and $a_{0}(n)+a_{1}(n)=$ $\frac{n}{n+2} a_{1}(n)$. Thus

$$
\left|D_{n}^{M, 1}(f, x)-f(x)\right| \leqslant 6(n+1) \omega\left(f, \sqrt{\frac{\delta_{n}(x)}{n}}\right) .
$$


Definition 4. 11]The Ditzian-Totik modulus of smoothness $\omega_{\varphi}^{2}(f, t)$ we shall use in our study is defined as: Let $f \in C[0,1]$, and $\varphi(x)=\sqrt{x(1-x)}$, then

$$
\omega_{\varphi}^{2}(f, t):=\sup _{0<h \leqslant t x+2 h \varphi(x) \geqslant 0} \sup _{h \varphi \vec{\Delta}^{2}}^{2} f(t) \|,
$$

where the second order forward difference of the function $f$ at a point $x$ is defined by

$$
\vec{\Delta}_{h \varphi(x)}^{2} f(x):=\left\{\begin{array}{l}
\sum_{j=0}^{2}(-1)^{2-j}\left(\begin{array}{l}
2 \\
j
\end{array}\right) f(x+j h \varphi(x)), \quad \text { if } x, x+2 h \varphi(x) \in[0,1] \\
0, \quad \text { otherwise. }
\end{array}\right.
$$

Definition 5. [11] The Peetre's K-functional corresponding to $\omega_{\varphi}^{2}(f, t)$ is defined as

$$
K_{2, \varphi}\left(f, t^{2}\right):=\inf _{g \in W_{2}}\left\{\|f-g\|+t^{2} \| \varphi^{2} g^{\prime \prime}\right\},
$$

where the class $W_{2}$ is given by $\left\{g:\left\|\varphi^{2} g^{\prime \prime}\right\|<\infty, g^{\prime} \in A C_{l o c}(0,1)\right\}$. Here, $A C_{l o c}(0,1)$ stands for the class of locally absolutely continuous functions on $(0,1)$.

Theorem 4. [11] There holds the equivalence

$$
\omega_{\varphi}^{2}(f, t) \sim K_{2, \varphi}\left(f, t^{2}\right) .
$$

Theorem 5. Let $f \in C[0,1]$ and let the operator $D_{n}^{M, 1}(f, x)$ be defined by 3.1). If $(n+2) a_{0}(n)+2 a_{1}(n)=0$, then there exists an absolute constant $C>0$ such that

$$
\left|D_{n}^{M, 1}(f, x)-f(x)\right| \leqslant C K_{2, \varphi}\left(f, n^{-2}\right) .
$$

Proof. We choose (see [11]) $g \in C^{3}[0,1]$ such that $g^{\prime \prime \prime}$ is locally absolutely continuous in $(0,1)$ and

$$
\|f-g\| \sim K_{2, \varphi}\left(f, \frac{\left|a_{0}(n)+a_{1}(n)\right|}{n^{2}}\right) .
$$

Now by linearity of $D_{n}^{M, 1}$ we can write

$$
\left|D_{n}^{M, 1}(f, x)-f(x)\right| \leqslant 2\|f-g\|+\left|D_{n}^{M, 1}(g, x)-g(x)\right| .
$$

By Taylor's formula

$$
g(t)-g(x)=\sum_{i=1}^{3} \frac{1}{i !}(t-x)^{i} g^{i}(x)+\frac{1}{3 !} \int_{x}^{t}(t-u)^{3} g^{(i v)}(u) d u .
$$

An application of $D_{n}^{M, 1}$ to both sides yields

$$
\begin{aligned}
D_{n}^{M, 1}(g, x)-g(x) & =\sum_{i=2}^{3} \frac{1}{i !} D_{n}^{M, 1}\left((t-x)^{i}, x\right) g^{i}(x)+\frac{1}{3 !} D_{n}^{M, 1}\left(\int_{x}^{t}(t-u)^{3} g^{(i v)}(u) d u, x\right) \\
& =\sum_{i=2}^{3} \frac{1}{i !} D_{n}^{M, 1}\left((t-x)^{i}, x\right) g^{i}(x)+\frac{1}{3 !}\left(R_{1}+R_{2}\right)
\end{aligned}
$$

where

$$
R_{1}=A_{n}\left(\int_{x}^{t}(t-u)^{3} g^{(i v)}(u) d u, x\right)
$$

and

$$
R_{2}=B_{n}\left(\int_{x}^{t}(t-u)^{3} g^{(i v)}(u) d u, x\right)
$$

Since, $D_{n}^{M, 1}(1, x)=1, D_{n}^{M, 1}\left(e_{1}, x\right)=x$,

$$
\begin{gathered}
D_{n}^{M, 1}\left(\left(e_{1}-x\right)^{2}, x\right)=\frac{2\left(1+n(n+5) \varphi^{2}(x)\right)}{n(n+2)(n+3)} \leqslant C \frac{\varphi^{2}(x)}{n}+o\left(\frac{1}{n}\right), \\
D_{n}^{M, 1}\left(\left(e_{1}-x\right)^{3}, x\right)=\frac{6(1-2 x)\left(n(n+9) \varphi^{2}(x)+2\right)}{n(n+2)(n+3)(n+4)} \leqslant C \frac{\varphi^{2}(x)}{n^{2}}+o\left(\frac{1}{n}\right), \\
\left|D_{n}^{M, 1}(g, x)-g(x)\right| \leqslant C\left(\frac{\varphi^{2}(x)\left|g^{\prime \prime}(x)\right|}{n^{2}}+\frac{\varphi^{2}(x)\left|g^{\prime \prime \prime}(x)\right|}{n^{2}}\right)+\left|R_{1}\right|+\left|R_{2}\right|+o\left(\frac{1}{n}\right) .
\end{gathered}
$$


By Lemma 5.3 of [],

$$
\left|\int_{x}^{t}(t-u)^{3} g^{(i v)}(u) d u\right| \leqslant\left|\frac{(t-x)^{3}}{\left(\varphi^{2}(x)+n^{-1}\right)^{2}} \int_{x}^{t}\left(\varphi^{2}(u)+\frac{1}{n}\right)^{2}\right| g^{(i v)}(u)|d u| .
$$

Therefore,

$$
\begin{aligned}
\left|R_{1}\right| & \leqslant \frac{1}{6} \frac{\left|a_{0}(n)+a_{1}(n)\right|}{\left(\varphi^{2}(x)+\frac{1}{n}\right)^{2}}\left(N\left(a_{0}(n), a_{1}(n)\right) \sum_{k=0}^{n} p_{n, k}(x) \int_{0}^{1}\left(\int_{x}^{t}\left(\varphi^{2}(u)+\frac{1}{n}\right)^{2}\left|g^{(i v)}(u)\right| d u\right)\right. \\
& \left.\times(t-x)^{4} p_{n-1, k}(t) \mathrm{d} t\right) \\
& \leqslant \frac{1}{6} \frac{\left|a_{0}(n)+a_{1}(n)\right|}{\left(\varphi^{2}(x)+\frac{1}{n}\right)^{2}}\|H(., .)\|\left(N\left(a_{0}(n), a_{1}(n)\right) \sum_{k=0}^{n} p_{n, k}(x) \int_{0}^{1}(t-x)^{4} p_{n-1, k}(t) \mathrm{d} t\right) \\
& \leqslant C \frac{\left|a_{0}(n)+a_{1}(n)\right|}{\left(\varphi^{2}(x)+\frac{1}{n}\right)^{2}}\|H\|\left(\frac{\varphi^{4}(x)}{n^{2}}+o\left(\frac{1}{n}\right)\right) \\
& \leqslant C \frac{\left|a_{0}(n)+a_{1}(n)\right|}{n^{2}}\left\|\varphi^{4} g^{(i v)}\right\| .
\end{aligned}
$$

Here we have used the inequality, $\|H\| \leqslant C\left\|\left(\varphi^{2}(x)+\frac{1}{n^{2}}\right)^{2} g^{(i v)}\right\|$.

Similarly,

$$
\left|R_{2}\right|=\left|B_{n}\left(\int_{x}^{t}(t-u)^{3} g^{(i v)}(u) d u, x\right)\right| \leqslant C \frac{\left|a_{0}(n)+a_{1}(n)\right|}{n^{2}}\left\|\varphi^{4} g^{(i v)}\right\| .
$$

Combining the estimates for $\left|R_{1}\right|,\left|R_{2}\right|$

$$
\left|D_{n}^{M, 1}(f, x)-f(x)\right| \leqslant C\left(\|f-g\|+\frac{\left\|\varphi^{2} g^{\prime \prime}\right\|}{n}+\frac{\left\|\varphi^{2} g^{\prime \prime \prime}\right\|}{n}+\frac{\left|a_{0}(n)+a_{1}\right|}{n^{2}}\left\|\varphi^{4} g^{(i v)}\right\|\right)
$$

Taking infimum over all $g \in W$, we get

$$
\left|D_{n}^{M, 1}(f, .)-f(.)\right| \leqslant C K_{2, \varphi}\left(f, \frac{\left|a_{0}(n)+a_{1}(n)\right|}{n^{2}}\right) \leqslant C \omega_{\varphi}^{4}\left(f, \frac{\sqrt[4]{\left|a_{0}(n)+a_{1}(n)\right|}}{n^{1 / 2}}\right)
$$

Remark 2. Let $a_{0}(n)$ and $a_{1}(n)$ be bounded sequences, and if $f \in C^{3}[0,1]$, then by repeated application of the inequality $\omega_{k+1}(f ; \delta) \leqslant \delta \omega_{k}\left(f^{\prime} ; \delta\right)$ there follows that

$$
\left|D_{n}^{M, 1}(f, .)-f(.)\right| \leqslant C \frac{\sqrt[4]{\left|a_{0}(n)+a_{1}(n)\right|^{3}}}{n^{3 / 2}} \omega_{\varphi}\left(f^{\prime \prime \prime}, \frac{\sqrt[4]{\left|a_{0}(n)+a_{1}(n)\right|}}{n^{1 / 2}}\right)
$$

Further, if $f \in C^{4}[0,1]$, then by $\omega_{k}(f ; \delta) \leqslant \delta^{k}\left\|f^{(i v)}\right\|$

$$
\left|D_{n}^{M, 1}(f, .)-f(.)\right| \leqslant C \frac{\left|a_{0}(n)+a_{1}(n)\right|}{n} .
$$

For a twice differentiable function $f$ we have following Voronovskaya type asymptotic result.

Theorem 6. Let $f \in C[0,1]$ be such that $f^{\prime \prime}(x)$ exists at $x$. Then, the limit

$$
\begin{aligned}
\lim _{n \rightarrow \infty}(n+2)\left(D_{n}^{M, 1}(f, x)-f(x)\right) & =\lim _{n \rightarrow \infty} \frac{(1-2 x)\left(a_{0}(n)(n+2)+2 a_{1}(n)\right)}{\left(2 a_{0}(n)(n+1)+a_{1}(n)(n+2)\right)} f^{\prime}(x) \\
& -\lim _{n \rightarrow \infty}\left(\frac{a_{0}(n)(n+3)\left(2(n-1) x^{2}-2(n-1) x-1\right)}{(n+3)\left(2 a_{0}(n)(n+1)+a_{1}(n)(n+2)\right)}\right. \\
& \left.+\frac{a_{1}(n)\left(\left(n^{2}+7 n-6\right) x^{2}-\left(n^{2}+7 n-6\right) x-3\right)}{(n+3)\left(2 a_{0}(n)(n+1)+a_{1}(n)(n+2)\right)}\right) f^{\prime \prime}(x)
\end{aligned}
$$

holds true. 
Proof. Applying $D_{n}^{M, 1}(f, x)$ to the expansion

$$
f(t)-f(x)=(t-x) f^{\prime}(x)+\frac{(t-x)^{2}}{2} f^{\prime \prime}(x)+(t-x)^{2} h(t, x)
$$

we get

$$
\begin{aligned}
D_{n}^{M, 1}(f, x)-f(x) & \left.=D_{n}^{M, 1}((t-x), x) f^{\prime}(x)+\frac{1}{2} D_{n}^{M, 1}\left((t-x)^{2}, x\right)\right) f^{\prime \prime}(x) \\
& +D_{n}^{M, 1}\left((t-x)^{2} h(t, x), x\right),
\end{aligned}
$$

where $h(t, x)$ is continuous function such that $\lim _{t \rightarrow x} h(t, x)=0$. By direct calculations we have

$$
D_{n}^{M, 1}((t-x), x)=\frac{(1-2 x)\left(a_{0}(n)(n+2)+2 a_{1}(n)\right)}{(n+2)\left(2 a_{0}(n)(n+1)+a_{1}(n)(n+2)\right)}
$$

and

$$
\begin{aligned}
D_{n}^{M, 1}\left((t-x)^{2}, x\right) & =-2\left(\frac{a_{0}(n)(n+3)\left(2(n-1) x^{2}-2(n-1) x-1\right)}{(n+2)(n+3)\left(2 a_{0}(n)(n+1)+a_{1}(n)(n+2)\right)}\right. \\
& \left.+\frac{a_{1}(n)\left(\left(n^{2}+7 n-6\right) x^{2}-\left(n^{2}+7 n-6\right) x-3\right)}{(n+2)(n+3)\left(2 a_{0}(n)(n+1)+a_{1}(n)(n+2)\right)}\right)
\end{aligned}
$$

We write

$$
D_{n}^{M, 1}\left((t-x)^{2} h(t, x), x\right)=A_{n}\left((t-x)^{2} h(t, x), x\right)+B_{n}\left((t-x)^{2} h(t, x), x\right) .
$$

By continuity, we can find a $\delta>0$ such that $|h(t, x)|<\epsilon$ for $|t-x|<\delta$. For the first term, we break the integral over $[0,1]$ into two sums which correspond to those $t$ and $x$ for which $|t-x|<\delta$ and $|t-x| \geqslant \delta$ respectively. Denote $(x-\delta, x+\delta)$ by $I_{\delta}$. By direct calculations

$$
A_{n}\left((t-x)^{2}, x\right)=\frac{2(1-x)\left(a_{0}(n)(n+3)((n-1) x+1)+a_{1}(n)\left((n-7) n x^{2}+(7 n-3) x+3\right)\right)}{(n+2)(n+3)\left(2 a_{0}(n)(n+1)+a_{1}(n)(n+2)\right)}
$$

and

$$
\begin{aligned}
A_{n}\left((t-x)^{4}, x\right) & =a\left(\frac{\left(n^{2}-13 n+2\right) x^{3}-\left(n^{2}-17 n+6\right) x^{2}-6(n-1) x-2}{(n+2)(n+3)(n+4)(2 a(n+1)+b(n+2))}\right) 12(x-1) \\
& +b\left(\frac{\left(-3\left(7 n^{2}-47 n+10\right) x^{2}-6(7 n-5) x-10\right)}{(n+2)(n+3)(n+4)(n+5)(2 a(n+1)+b(n+2))}\right. \\
& \left.+\frac{n\left(n^{2}-33 n+62\right) x^{4}-\left(n^{3}-52 n^{2}+161 n-10\right) x^{3}}{(n+2)(n+3)(n+4)(n+5)(2 a(n+1)+b(n+2))}\right) 12(x-1)
\end{aligned}
$$

$$
\begin{aligned}
& \left|A_{n}\left((t-x)^{2} h(t, x), x\right)\right| \\
& =\left|N\left(a_{0}(n), a_{1}(n)\right) \sum_{k=0}^{n} p_{n, k}(x)\left(\int_{I_{\delta}}+\int_{[0,1] \backslash I_{\delta}}\right) p_{n-1, k}(t)\left(a_{0}(n)+a_{1}(n) t\right)(t-x)^{2} h(t, x) \mathrm{d} t\right| \\
& =\leq\left|N\left(a_{0}(n), a_{1}(n)\right)\right| \int_{0}^{1} p_{n-1, k}(t)\left(\epsilon+\frac{M}{\delta^{2}}(t-x)^{2}\right)\left(a_{0}(n)+a_{1}(n) t\right)(t-x)^{2} \mathrm{~d} t
\end{aligned}
$$

If $a_{0}(n)$ and $a_{1}(n)$ are such that $2 a_{0}(n)(n+1)+a_{1}(n)(n+2)$ is positive, then

$$
\left|A_{n}\left((t-x)^{2} h(t, x), x\right)\right| \leq \epsilon A_{n}\left((t-x)^{2}, x\right)+\frac{M}{\delta^{2}} A_{n}\left((t-x)^{4}, x\right) .
$$

Case 1. If $a_{0}(n)$ and $a_{1}(n)$ are positive and convergent, then $\frac{M}{\delta^{2}} A_{n}\left((t-x)^{4}, x\right)$ becomes arbitrarily small for sufficiently large values of $n$.

Case 2. If $a_{0}(n)$ and $a_{1}(n)$ are positive and such that $2 a_{0}(n)(n+1)+a_{1}(n)(n+2)$ remains bounded for all values of $n$, then again $\frac{M}{\delta^{2}} A_{n}\left((t-x)^{4}, x\right)$ tends to zero as $n$ tends to infinity. Hence, in these two cases $A_{n}\left((t-x)^{2} h(t, x), x\right)$ 
tends to zero as $n$ tends to infinity. By a similar argument $B_{n}\left((t-x)^{2} h(t, x), x\right) \rightarrow 0$ as $n$ tends to infinity. Case 3. If $a_{0}(n)$ and $a_{1}(n)$ are convergent and such that $2 a_{0}(n)(n+1)+a_{1}(n)(n+2) \neq 0$ then

$$
\begin{aligned}
& \left|A_{n}\left((t-x)^{2} h(t, x), x\right)\right| \\
& =\left|N\left(a_{0}(n), a_{1}(n)\right)\right|\left|a_{0}(n)+a_{1}(n)\right| \sum_{k=0}^{n} p_{n, k}(x) \int_{0}^{1} p_{n-1, k}(t)\left(\epsilon(t-x)^{2}+\frac{M}{\delta^{2}}(t-x)^{4}\right) \mathrm{d} t \\
& \leq\left|N\left(a_{0}(n), a_{1}(n)\right)\right|\left|a_{0}(n)+a_{1}(n)\right| \times \\
& 2(1-x)\left(\epsilon \frac{\left(a_{0}(n)(n+3)((n-1) x+1)+a_{1}(n)\left((n-7) n x^{2}+(7 n-3) x+3\right)\right)}{n(n+1)(n+2)(n+3)}\right) \\
& -6 a_{0}(n) \frac{M}{\delta^{2}}\left(\frac{\left(n^{2}-13 n+2\right) x^{3}-\left(n^{2}-17 n+6\right) x^{2}-6(n-1) x-2}{n(n+1)(n+2)(n+3)(n+4)}\right) \\
& -6 a_{1}(n) \frac{M}{\delta^{2}}\left(\frac{\left(-3\left(7 n^{2}-47 n+10\right) x^{2}-6(7 n-5) x-10\right)}{n(n+1)(n+2)(n+3)(n+4)(n+5)}\right. \\
& \left.\left.+\frac{n\left(n^{2}-33 n+62\right) x^{4}-\left(n^{3}-52 n^{2}+161 n-10\right) x^{3}}{n(n+1)(n+2)(n+3)(n+4)(n+5)}\right)\right)
\end{aligned}
$$

Again $A_{n}\left((t-x)^{2} h(t, x), x\right)$ tends to zero as $n$ tends to infinity. Similar arguments hold true for $B_{n}\left((t-x)^{2} h(t, x), x\right)$. This completes the proof.

Corollary 4. Setting $a_{0}(n)=-2 a_{1}(n) /(n+2)$ and $a_{1}(n)$ is not a null sequence, then

$$
\lim _{n \rightarrow \infty}(n+2)\left(D_{n}^{M, 1}(f, x)-f(x)\right)=\varphi^{2}(x) f^{\prime \prime}(x) .
$$

Corollary 5. When $a_{0}(n)=1$ and $a_{1}(n)=-1$ we get Voronovskaya theorem for Bernstein-Durrmeyer operators (2.1) as follows

$$
\lim _{n \rightarrow \infty}(n+2)\left(D_{n}^{M, 1}(f, x)-f(x)\right)=(1-2 x) f^{\prime}(x)+\varphi^{2}(x) f^{\prime \prime}(x)
$$

\section{BÉzier VARIANt of the operator $D_{n}^{M, 1}(f ; x)$}

We further extend the error estimates by the Bézier Variant of the operator $D_{n}^{M, 1}(f ; x)$ for a larger class, namely the class of functions of bounded variation. For $\mu \geq 1$ and $f \in L_{B}[0,1]$, we define Bézier variant of the operator $D_{n}^{M, 1}(f ; x)$ as

$$
D_{n, \mu}^{M, 1}(f ; x):=N\left(a_{0}(n), a_{1}(n)\right) \sum_{k=0}^{n} Q_{n, k}^{(\mu)}(x) \int_{0}^{1} p_{n, k}^{M, 1}(u) f(u) \mathrm{d} u,
$$

where

$$
Q_{n, k}^{(\mu)}(x)=\left(J_{n, k}(x)\right)^{\mu}-\left(J_{n, k+1}(x)\right)^{\mu}, J_{n, k}(x)=\sum_{j=k}^{n} p_{n, j}(x) .
$$

For $\mu=1$, this family of Bézier operators yield the operators (3.1). Let

$$
R_{n, \mu}(x, u)=N\left(a_{0}(n), a_{1}(n)\right) \sum_{k=0}^{n} Q_{n, k}^{(\mu)}(x) p_{n, k}^{M, 1}(u) .
$$

Then $D_{n, \mu}^{M, 1}(f ; x):=\int_{0}^{1} R_{n, \mu}(x, u) f(u) \mathrm{d} u$.

Lemma 2. If $f \in C[0,1]$ then, $\left\|D_{n}^{M, 1}(f)\right\| \leq\|f\|$, where $\|$.$\| denotes the sup-norm on [0,1]$.

Lemma 3. If $f \in C[0,1]$ then, $\left\|D_{n, \mu}^{M, 1}(f)\right\| \leq \mu\|f\|$.

Proof. Using the inequality $\left|a^{\mu}-b^{\mu}\right| \leq \mu|a-b|$ with $0 \leq a, b \leq 1, \mu \geq 1$ and definition of $D_{n, \mu}^{M, 1}(f)$, we get for $\mu \geq 1$

$$
0<\left[\left(J_{n, k}(x)\right)^{\mu}-\left(J_{n, k+1}(x)\right)^{\mu}\right] \leq \mu\left[J_{n, k}(x)-J_{n, k+1}(x)\right]=\mu p_{n, k}(x)
$$

Applying $D_{n, \mu}^{M, 1}(f ; x)$ and Lemma 3 , we get

$$
\left\|D_{n, \mu}^{M, 1}(f)\right\| \leq \mu\left\|D_{n}^{M, 1}(f)\right\| \leq \mu\|f\| .
$$


Now, we derive a direct result for Bézier operators in terms of Ditzian-Totik modulus of smoothness $\omega_{\varphi}(f, t)$.

Theorem 7. Let $\varphi(x)=\sqrt{x(1-x)}, \mu \geq 1$ and $x \in[0,1]$. If $f \in C[0,1]$ then

$$
\left|D_{n, \mu}^{M, 1}(f ; x)-f(x)\right| \leq C \omega_{\varphi}\left(f, \sqrt{\frac{1}{n+2}}\right),
$$

where $C$ is any absolute constant.

Proof. We know that $g(t)=g(x)+\int_{x}^{t} g^{\prime}(u) \mathrm{d} u$. So

$$
\left|D_{n, \mu}^{M, 1}(g ; x)-g(x)\right|=\left|D_{n, \mu}^{M, 1}\left(\int_{x}^{t} g^{\prime}(u) \mathrm{d} u, x\right)\right| .
$$

For any $x, t \in(0,1)$, we have

$$
\left|\int_{x}^{t} g^{\prime}(u) \mathrm{d} u\right| \leq\left\|\varphi g^{\prime}\right\|\left|\int_{x}^{t} \frac{1}{\varphi(u)} \mathrm{d} u\right|
$$

Therefore,

$$
\begin{aligned}
\left|\int_{x}^{t} \frac{1}{\varphi(u)} \mathrm{d} u\right| & =\left|\int_{x}^{t} \frac{1}{\sqrt{u(1-u)}} \mathrm{d} u\right| \\
& \leq\left|\int_{x}^{t}\left(\frac{1}{\sqrt{u}}+\frac{1}{\sqrt{1-u}}\right) \mathrm{d} u\right| \\
& \leq 2(|\sqrt{t}-\sqrt{x}|+|\sqrt{1-t}-\sqrt{1-x}|) \\
& =2|t-x|\left(\frac{1}{\sqrt{t}+\sqrt{x}}+\frac{1}{\sqrt{1-t}+\sqrt{1-x}}\right) \\
& <2|t-x|\left(\frac{1}{\sqrt{x}}+\frac{1}{\sqrt{1-x}}\right) \\
& <\frac{2 \sqrt{2}|t-x|}{\varphi(x)} .
\end{aligned}
$$

Combining 6.3)-6.5) and using Cauchy-Schwarz inequality, we have that

$$
\begin{aligned}
\left|D_{n, \mu}^{M, 1}(g ; x)-g(x)\right| & \leq 2 \sqrt{2}\left\|\varphi g^{\prime}\right\| \varphi^{-1}(x) D_{n, \mu}^{M, 1}(|t-x| ; x) \\
& \leq 2 \sqrt{2}\left\|\varphi g^{\prime}\right\| \varphi^{-1}(x)\left(D_{n, \mu}^{M, 1}\left((t-x)^{2} ; x\right)\right)^{1 / 2} .
\end{aligned}
$$

Now using inequality 4.3 , we obtain

$$
\left|D_{n, \mu}^{M, 1}(g ; x)-g(x)\right|<C \sqrt{\frac{1}{(n+2)}}\left\|\varphi g^{\prime}\right\| .
$$

Using Lemma 1 and inequality (6.6), we obtain

$$
\begin{aligned}
\left|D_{n, \mu}^{M, 1}(f ; x)-f\right| & \leq\left|D_{n, \mu}^{M, 1}(f-g ; x)\right|+|f-g|+\left|D_{n, \mu}^{M, 1}(g ; x)-g(x)\right| \\
& \leq C\left(\|f-g\|+\sqrt{\frac{1}{(n+2)}}\left\|\varphi g^{\prime}\right\|\right) .
\end{aligned}
$$

Taking infimum on both sides of 6.7 over all $g \in W_{\phi}^{2}$, we reach to

$$
\left|D_{n, \mu}^{M, 1}(f ; x)-f\right| \leq C K_{\varphi}\left(f, \sqrt{\frac{1}{(n+2)}}\right) .
$$

Using relation $K_{\varphi}(f, t) \sim \omega_{\varphi}(f, t)$, we get the required result. 
Ozarslan and Aktuglu [28] is considered the Lipschitz-type space with two parameters $\alpha_{1} \geq 0, \alpha_{2}>0$, which is defined as

$$
\operatorname{Lip}_{M}^{\left(\alpha_{1}, \alpha_{2}\right)}(\zeta)=\left\{f \in C[0,1]:|f(t)-f(x)| \leq C_{0} \frac{|t-x|^{\zeta}}{\left(t+\alpha_{1} x^{2}+\alpha_{2} x\right)^{\zeta / 2}}: t \in[0,1], x \in(0,1)\right\}
$$

where $0<\zeta \leq 1$, and $C_{0}$ is any absolute constant.

Theorem 8. Let $f \in \operatorname{Lip}_{M}^{\left(\alpha_{1}, \alpha_{2}\right)}(\zeta)$. Then for all $x \in(0,1]$, there holds:

$$
\left|D_{n, \mu}^{M, 1}(f ; x)-f\right| \leq C\left(\frac{\mu \varphi^{2}(x)}{(n+2)\left(\alpha_{1} x^{2}+\alpha_{2} x\right)}\right)^{\frac{\zeta}{2}}
$$

where $C$ is any absolute constant.

Proof. Using Lemma 1 and 6.2 and Hölder's inequality with $p=\frac{2}{\zeta}$ and $p=\frac{2}{2-\zeta}$, we get

$$
\begin{aligned}
& \left|D_{n, \mu}^{M, 1}(f ; x)-f(x)\right| \\
& \leq N\left(a_{0}(n), a_{1}(n)\right) \sum_{k=0}^{n} Q_{n, k}^{(\mu)}(x) \int_{0}^{1} p_{n, k}^{M, 1}(u)|f(u)-f(x)| \mathrm{d} u \\
& \leq N\left(a_{0}(n), a_{1}(n)\right) \sum_{k=0}^{n} Q_{n, k}^{(\mu)}(x)\left(\int_{0}^{1} p_{n, k}^{M, 1}(u)|f(u)-f(x)|^{\frac{2}{\zeta}} \mathrm{d} u\right)^{\frac{\zeta}{2}} \\
& \leq\left(N\left(a_{0}(n), a_{1}(n)\right) \sum_{k=0}^{n} Q_{n, k}^{(\mu)}(x) \int_{0}^{1} p_{n, k}^{M, 1}(u)|f(u)-f(x)|^{\frac{2}{\zeta}} \mathrm{d} u\right)^{\frac{\zeta}{2}} \times \\
& \left(N\left(a_{0}(n), a_{1}(n)\right) \sum_{k=0}^{n} Q_{n, k}^{(\mu)}(x) \int_{0}^{1} p_{n, k}^{M, 1}(u) \mathrm{d} u\right)^{\frac{2-\zeta}{2}} \\
& =\left(N\left(a_{0}(n), a_{1}(n)\right) \sum_{k=0}^{n} Q_{n, k}^{(\mu)}(x) \int_{0}^{1} p_{n, k}^{M, 1}(u)|f(u)-f(x)|^{\frac{2}{\zeta}} \mathrm{d} u\right)^{\frac{\zeta}{2}} \\
& \leq C\left(N\left(a_{0}(n), a_{1}(n)\right) \sum_{k=0}^{n} Q_{n, k}^{(\mu)}(x) \int_{0}^{1} p_{n, k}^{M, 1}(u) \frac{(u-x)^{2}}{\left(u+\alpha_{1} x^{2}+\alpha_{2} x\right)} \mathrm{d} u\right)^{\frac{\zeta}{2}} \\
& \leq \frac{C}{\left(\alpha_{1} x^{2}+\alpha_{2} x\right)^{\frac{\zeta}{2}}}\left(N\left(a_{0}(n), a_{1}(n)\right) \sum_{k=0}^{n} Q_{n, k}^{(\mu)}(x) \int_{0}^{1} p_{n, k}(u)(u-x)^{2} \mathrm{~d} u\right)^{\frac{\zeta}{2}} \\
& \leq \frac{C}{\left(\alpha_{1} x^{2}+\alpha_{2} x\right)^{\frac{\zeta}{2}}}\left[D_{n, \mu}^{M, 1}\left((u-x)^{2} ; x\right)\right]^{\frac{\zeta}{2}} \\
& \leq C\left(\frac{\mu \varphi^{2}(x)}{(n+2)\left(\alpha_{1} x^{2}+\alpha_{2} x\right)}\right)^{\frac{\zeta}{2}} \text {. }
\end{aligned}
$$

Hence, the desired result follows.

If we define

$$
\kappa_{n, \mu}(x, y)=\int_{0}^{y} R_{n, \mu}(x, t) \mathrm{d} t
$$

Then, it is obvious that $\kappa_{n, \mu}(x, 1)=1$.

Lemma 4. Let $x \in(0,1)$ and $C>2$ then for sufficiently large $n$ we have

$$
\begin{gathered}
\kappa_{n, \mu}(x, y)=\int_{0}^{y} R_{n, \mu}(x, t) d t \leq \frac{C \mu x(1-x)}{n(x-y)^{2}}, \quad 0<y<x \\
1-\kappa_{n, \mu}(x, z)=\int_{z}^{1} R_{n, \mu}(x, t) d t \leq \frac{C \mu x(1-x)}{n(z-x)^{2}}, \quad x<z<1 .
\end{gathered}
$$


Remark 3. We have

$$
\begin{aligned}
\kappa_{n, \mu}(x, y) & =\int_{0}^{y} R_{n, \mu}(x, t) d t \leq \int_{0}^{y} R_{n, \mu}(x, t) \frac{(t-x)^{2}}{(y-x)^{2}} d t \\
& =\frac{D_{n, \mu}^{M, 1}\left((t-x)^{2} ; x\right)}{(y-x)^{2}} \leq \frac{\mu D_{n}^{M, 1}\left((t-x)^{2} ; x\right)}{(y-x)^{2}} \leq \frac{C \mu}{n+2} \cdot \frac{\varphi^{2}(x)}{(y-x)^{2}} .
\end{aligned}
$$

Now, we discuss the approximation properties of functions having derivative of bounded variation on [0, 1$]$. Let $\mathbb{B}[0,1]$ denote the class of differentiable functions $g$ defined on $[0,1]$, whose derivative $g^{\prime}$ is of bounded variation on $[0,1]$. The functions $g \in \mathbb{B}[0,1]$ is expressed as $g(x)=\int_{0}^{x} h(t) d t+g(0)$, where $h \in \mathbb{B}[0,1]$, i.e., $h$ is a function of bounded variation on $[0,1]$.

Theorem 9. Let $f \in \mathbb{B}[0,1]$ then for $\mu \geq 1,0<x<1$ a and sufficiently large $n$ we have

$$
\begin{aligned}
& \left|D_{n, \mu}^{M, 1}(f ; x)-f(x)\right| \leq\left(\frac{1}{\mu+1}\left|f^{\prime}(x+)+\mu f^{\prime}(x-)\right|+\left|f^{\prime}(x+)-f^{\prime}(x-)\right|\right) \frac{\mu}{n+2} \varphi^{2}(x) \\
& +\frac{\mu}{n+2} \cdot \frac{\varphi^{2}(x)}{x^{2}} \sum_{k=1}^{\sqrt{n}}\left(\bigvee_{x-\frac{x}{k}}^{x}\left(f^{\prime}\right)_{x}\right)+\frac{x}{\sqrt{n}}\left(\bigvee_{x}^{x+\frac{1-x}{\sqrt{n}}}\left(f^{\prime}\right)_{x}\right)\left(\bigvee_{x-\frac{x}{k}}^{x}\left(f^{\prime}\right)_{x}\right) \\
& +\frac{\mu}{n+2} \cdot \frac{\varphi^{2}(x)}{1-x} \sum_{k=1}^{\sqrt{n}}\left(\bigvee_{x}^{x+\frac{1-x}{k}}\left(f^{\prime}\right)_{x}\right)+\frac{1-x}{\sqrt{n}}\left(\bigvee_{x}^{x+\frac{1-x}{\sqrt{n}}}\left(f^{\prime}\right)_{x}\right) \text {. } \\
& \left(f^{\prime}\right)_{x}(t)= \begin{cases}f^{\prime}(t)-f^{\prime}(x-), & 0 \leq t<x \\
0, & t=x \\
f^{\prime}(t)-f^{\prime}(x+), & x<t \leq 1\end{cases}
\end{aligned}
$$

Proof. As we know that $D_{n, \mu}^{M, 1}(1 ; x)=1$. Therefore, we have

$$
\begin{aligned}
D_{n, \mu}^{M, 1}(f ; x)-f(x) & =\int_{0}^{1} R_{n, \mu}(x, u)(f(u)-f(x)) \mathrm{d} u \\
& =\int_{0}^{1} R_{n, \mu}(x, u) \int_{x}^{u} f^{\prime}(t) d t \mathrm{~d} u
\end{aligned}
$$

Since $f \in \mathbb{B}[0,1]$, we may write

$$
\begin{aligned}
f^{\prime}(t) & =\frac{f^{\prime}(x+)+\mu f^{\prime}(x-)}{\mu+1}+\left(f^{\prime}\right)_{x}(t)+\frac{f^{\prime}(x+)-f^{\prime}(x-)}{2}\left(\operatorname{sign}(t-x)+\frac{\mu-1}{\mu+1}\right) \\
& +\delta_{x}(t)\left(f^{\prime}(t)-\frac{f^{\prime}(x+)+\mu f^{\prime}(x-)}{2}\right)
\end{aligned}
$$

where

$$
\operatorname{sign}(t)= \begin{cases}1, & t>0 \\ 0, & t=0 \\ -1, & t<0\end{cases}
$$

and

$$
\delta_{x}(t)= \begin{cases}1, & t=x \\ 0, & t \neq x\end{cases}
$$

Putting the value of $f^{\prime}(t)$ form 6.9 in 6.8 , we get estimates corresponding to four terms of 6.9$)$, say $I_{1}, I_{2}, I_{3}$ and $I_{4}$ respectively. Obviously,

$$
I_{4}=\int_{0}^{1}\left(\int_{x}^{u}\left(f^{\prime}(t)-\frac{f^{\prime}(x+)+\mu f^{\prime}(x-)}{2}\right) \delta_{x}(t) d t\right) R_{n, \mu}(x, u) \mathrm{d} u=0 .
$$


Now, Using Cauchy's Schwarz inequality and Lemma 4, we obtain

$$
\begin{aligned}
I_{1}=\int_{0}^{1}\left(\int_{x}^{u} \frac{f^{\prime}(x+)+\mu f^{\prime}(x-)}{\mu+1} d t\right) R_{n, \mu}(x, u) \mathrm{d} u & =\frac{f^{\prime}(x+)+\mu f^{\prime}(x-)}{\mu+1} \int_{0}^{1}(u-x) R_{n, \mu}(x, u) \mathrm{d} u \\
& =\frac{f^{\prime}(x+)+\mu f^{\prime}(x-)}{\mu+1} D_{n, \mu}^{M, 1}((u-x) ; x) \\
& \leq \frac{1}{\sqrt{n+2}} \frac{f^{\prime}(x+)+\mu f^{\prime}(x-)}{\mu+1} \varphi(x) .
\end{aligned}
$$

Next, again using Cauchy's Schwarz inequality and Lemma 4, we obtain

$$
\begin{aligned}
I_{3} & =\int_{0}^{1}\left(\int_{x}^{u}\left(\frac{f^{\prime}(x+)-f^{\prime}(x-)}{2}\right)\left(\operatorname{sign}(t-x)+\frac{\mu-1}{\mu+1}\right) d t\right) R_{n, \mu}(x, u) \mathrm{d} u \\
& =\left(\frac{f^{\prime}(x+)-f^{\prime}(x-)}{2}\right)\left(-\int_{0}^{x}\left(\int_{u}^{x}\left(\operatorname{sign}(t-x)+\frac{\mu-1}{\mu+1}\right) d t\right) R_{n, \mu}(x, u) \mathrm{d} u\right. \\
& \left.+\int_{x}^{1}\left(\int_{x}^{u}\left(\operatorname{sign}_{\mu}(t-x)+\frac{\mu-1}{\mu+1}\right) d t\right) R_{n, \mu}(x, u) \mathrm{d} u\right) \\
& \leq\left|f^{\prime}(x+)-f^{\prime}(x-)\right| \int_{0}^{1}|u-x| R_{n, \mu}(x, u) \mathrm{d} u \\
& \leq\left|f^{\prime}(x+)-f^{\prime}(x-)\right| D_{n, \mu}^{M, 1}(|u-x| ; x) \\
& \leq \frac{C}{\sqrt{n+2}}\left|f^{\prime}(x+)-f^{\prime}(x-)\right| \varphi(x) .
\end{aligned}
$$

Now, we estimate $I_{2}$ as follows:

$$
\begin{aligned}
I_{2}=\int_{0}^{1}\left(\int_{x}^{u}\left(f^{\prime}\right)_{x}(t) d t\right) R_{n, \mu}(x, u) \mathrm{d} u & =\int_{0}^{x}\left(\int_{x}^{u}\left(f^{\prime}\right)_{x}(t) d t\right) R_{n, \mu}(x, u) \mathrm{d} u \\
& +\int_{x}^{1}\left(\int_{x}^{u}\left(f^{\prime}\right)_{x}(t) d t\right) R_{n, \mu}(x, u) \mathrm{d} u \\
& =I_{5}+I_{6}, \text { say. }
\end{aligned}
$$

Using Lemma 4 and definition of $\kappa_{n, \mu}(x, u)$, we may write

$$
I_{5}=\int_{0}^{x}\left(\int_{x}^{u}\left(f^{\prime}\right)_{x}(t) d t\right) \frac{d}{\mathrm{~d} u} \kappa_{n, \mu}(x, u) \mathrm{d} u
$$

Integrating by parts, we obtain

$$
\begin{aligned}
\left|I_{5}\right| & \leq \int_{0}^{x}\left|\left(f^{\prime}\right)_{x}(u)\right| \kappa_{n, \mu}(x, u) \mathrm{d} u \\
& \leq \int_{0}^{x-x / \sqrt{n}}\left|\left(f^{\prime}\right)_{x}(u)\right| \kappa_{n, \mu}(x, u) \mathrm{d} u+\int_{x-x / \sqrt{n}}^{x}\left|\left(f^{\prime}\right)_{x}(u)\right| \kappa_{n, \mu}(x, u) \mathrm{d} u \\
& =I_{7}+I_{8}, \text { say. }
\end{aligned}
$$


In view of facts $\left(f^{\prime}\right)_{x}(x)=0$ and $\kappa_{n, \mu}(x, u) \leq 1$, we get

$$
\begin{aligned}
I_{8} & =\int_{0}^{x}\left|\left(f^{\prime}\right)_{x}(u)-\left(f^{\prime}\right)_{x}(x)\right| \kappa_{n, \mu}(x, u) \mathrm{d} u \\
& \leq \int_{x-x / \sqrt{n}}^{x}\left(\bigvee_{u}^{x}\left(f^{\prime}\right)_{x}\right) \mathrm{d} u \\
& \leq\left(\bigvee_{u}^{x}\left(f^{\prime}\right)_{x}\right) \int_{x-x / \sqrt{n}}^{x} \mathrm{~d} u=\frac{x}{\sqrt{n}}\left(\bigvee_{u}^{x}\left(f^{\prime}\right)_{x}\right) .
\end{aligned}
$$

Using Lemma 4 definition of $\kappa_{n, \mu}(x, u)$, and transformation $u=x-\frac{x}{t}$ we may write

$$
\begin{aligned}
I_{7} & \leq \frac{C \mu}{n+2} \varphi^{2}(x) \int_{0}^{x-x / \sqrt{n}}\left|\left(f^{\prime}\right)_{x}(u)-\left(f^{\prime}\right)_{x}(x)\right| \frac{\mathrm{d} u}{(u-x)^{2}} \\
& \leq \frac{C \mu}{n+2} \varphi^{2}(x) \int_{0}^{x-x / \sqrt{n}}\left(\bigvee_{u}^{x}\left(f^{\prime}\right)_{x}\right) \frac{\mathrm{d} u}{(u-x)^{2}} \\
& \leq \frac{C \mu}{n+2} \frac{\varphi^{2}(x)}{x^{2}} \int_{1}^{\sqrt{n}}\left(\bigvee_{x-\frac{x}{t}}^{x}\left(f^{\prime}\right)_{x}\right) d t \\
& \leq \frac{C \mu}{n+2} \frac{\varphi^{2}(x)}{x^{2}} \sum_{k=1}^{|\sqrt{n}|}\left(\bigvee_{x-\frac{x}{t}}^{x}\left(f^{\prime}\right)_{x}\right) .
\end{aligned}
$$

Combining the estimates of $I_{7}$ and $I_{8}$, we have

$$
\left|I_{5}\right| \leq \frac{C \mu}{n+2} \frac{\varphi^{2}(x)}{x^{2}} \sum_{k=1}^{|\sqrt{n}|}\left(\bigvee_{x-\frac{x}{t}}^{x}\left(f^{\prime}\right)_{x}\right)+\frac{x}{\sqrt{n}}\left(\bigvee_{u}^{x}\left(f^{\prime}\right)_{x}\right)
$$


In order to estimate $I_{6}$, we use integration by parts, Lemma 4 and transformation $z=x+\frac{1-x}{\sqrt{n}}$. Therefore, we proceed as follows:

$$
\begin{aligned}
\left|I_{6}\right| & =\left|\int_{x}^{1}\left(\int_{x}^{u}\left(f^{\prime}\right)_{x}(t) d t\right) R_{n, \mu}(x, u) \mathrm{d} u\right| \\
& =\left|\int_{x}^{z}\left(\int_{x}^{u}\left(f^{\prime}\right)_{x}(t) d t\right) \frac{\partial}{\partial u}\left(1-\kappa_{n, \mu}(x, u)\right) \mathrm{d} u+\int_{z}^{1}\left(\int_{x}^{u}\left(f^{\prime}\right)_{x}(t) d t\right) \frac{\partial}{\partial u}\left(1-\kappa_{n, \mu}(x, u)\right) \mathrm{d} u\right| \\
& =\mid\left(\int_{x}^{u}\left(f^{\prime}\right)_{x}(t) d t\left(1-\kappa_{n, \mu}(x, u)\right)\right)_{x}^{z}-\int_{x}^{z}\left(f^{\prime}\right)_{x}(u)\left(1-\kappa_{n, \mu}(x, u)\right) \mathrm{d} u \\
& \left.+\mid \int_{x}^{u}\left(f^{\prime}\right)_{x}(t) d t\left(1-\kappa_{n, \mu}(x, u)\right)\right)_{z}^{1}-\int_{z}^{1}\left(f^{\prime}\right)_{x}(u)\left(1-\kappa_{n, \mu}(x, u)\right) \mathrm{d} u \mid \\
& =\left|\int_{x}^{z}\left(f^{\prime}\right)_{x}(u)\left(1-\kappa_{n, \mu}(x, u)\right) \mathrm{d} u+\int_{z}^{1}\left(f^{\prime}\right)_{x}(u)\left(1-\kappa_{n, \mu}(x, u)\right) \mathrm{d} u\right| \\
& \leq \frac{C \mu}{n+2} \cdot \varphi^{2}(x) \int_{z}^{1}\left(\bigvee_{x}^{u}\left(f^{\prime}\right)_{x}\right)(u-x)^{-2} \mathrm{~d} u+\int_{x}^{z}\left(\bigvee_{x}^{u}\left(f^{\prime}\right)_{x}\right) \mathrm{d} u \\
& \leq \frac{C \mu}{n+2} \cdot \varphi^{2}(x) \int_{x+\frac{1-x}{\sqrt{n}}}^{1}\left(\bigvee_{x}^{u}\left(f^{\prime}\right)_{x}\right)(u-x)^{-2} \mathrm{~d} u+\frac{1-x}{\sqrt{n}}\left(\begin{array}{l}
x+\frac{1-x}{\sqrt{n}} \\
\bigvee_{x}
\end{array}\left(f^{\prime}\right)_{x}\right)
\end{aligned}
$$

Now, substituting $t=\frac{1-x}{t-x}$, we get

$$
\begin{aligned}
\left|I_{6}\right| & \leq \frac{C \mu}{n+2} \cdot \varphi^{2}(x) \int_{1}^{\sqrt{n}}\left(\bigvee_{x}^{x+\frac{1-x}{t}}\left(f^{\prime}\right)_{x}\right)(1-x)^{-1} d t+\frac{1-x}{\sqrt{n}}\left(\bigvee_{x}^{x+\frac{1-x}{\sqrt{n}}}\left(f^{\prime}\right)_{x}\right) \\
& \leq \frac{C \mu}{n+2} \frac{\varphi^{2}(x)}{1-x} \sum_{k=1}^{\sqrt{n}}\left(\bigvee_{x}^{x+\frac{1-x}{k}}\left(f^{\prime}\right)_{x}\right)+\frac{1-x}{\sqrt{n}}\left(\bigvee_{x}^{x+\frac{1-x}{\sqrt{n}}}\left(f^{\prime}\right)_{x}\right) .
\end{aligned}
$$

Combining the estimates of $I_{1}-I_{8}$, we get the desired result. Hence the proof follows.

\section{Numerical ExPERIMENTS}

This part of the paper is presented to verify the theoretical results with some numerical examples. All computations are carried out by the Maple 15 software.

To do so and for the first step, we are interested in knowing the behavior of $p_{n, k}(x)$ and $p_{n, k}^{M, 1}(x)$ in $[0,1]$.

At first, it should be noted that in the rest of this paper we consider:

$$
a_{0}(n)=-\frac{2}{n}, a_{1}(n)=1+\frac{2}{n} .
$$

Comparison of the behavior of these polynomials is plotted in Fig. 1 with $n=5$ and $k=1: 1: 5$. It is easily observed form this figure that $p_{n, k}^{M, 1}(x)$ are negative for some values of $n$ and $k$.

For the second step, we need to approximate the interior integral in the new operator (2.1) as:

$$
\int_{0}^{1} p_{n, k}^{M, 1}(x) f(x) \mathrm{d} x=\sum_{r=0}^{N} w_{r} p_{n, k}^{M, 1}\left(x_{r}\right) f\left(x_{r}\right)+E_{N}^{n, k}(f),
$$

where $x_{r}$ and $w_{r}$ for $r=0,1,2, \cdots, N$ are the nodes and weights of the quadrature rule, respectively. We also note that $E_{N}^{n, k}(f)$ is known as error function 32. Therefore, the Modified-Bernstein-Durrmeyer operator 2.1] can be 

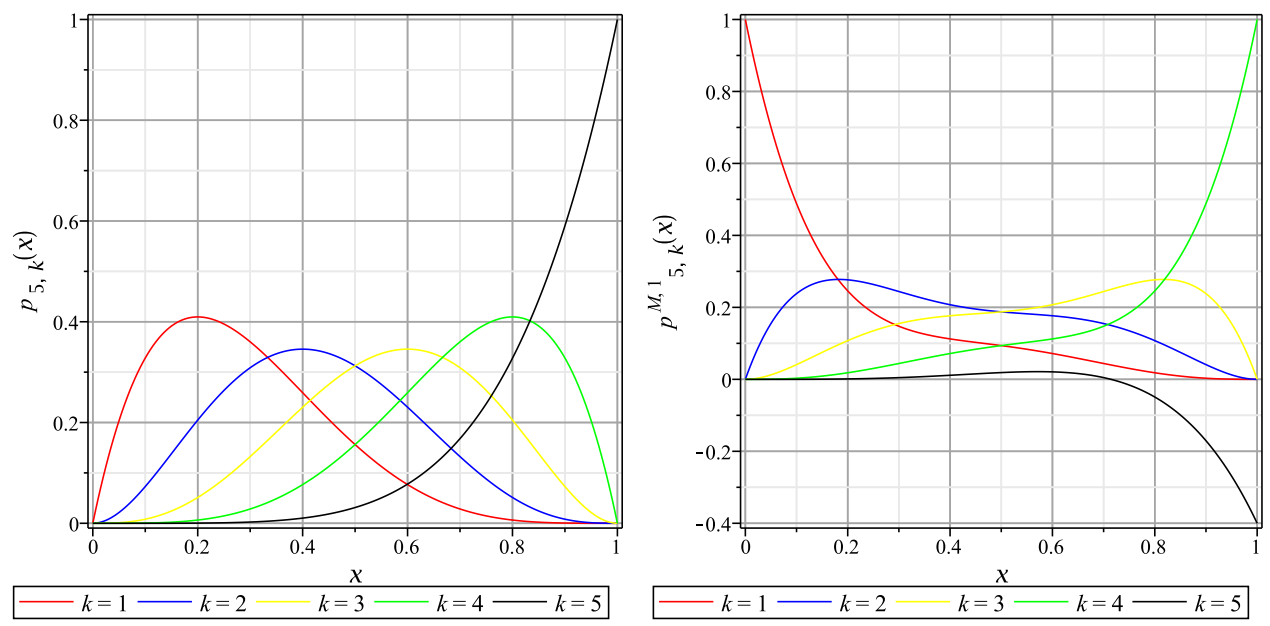

Figure 1. Comparison of the behavior of $p_{n, k}(x)$ and $p_{n, k}^{M, 1}(x)$ for $n=5$ and some values of $k$.

written as follows:

$$
D_{n}^{M, 1}(f ; x):=\frac{n(n+1)}{\left(2 a_{0}(n)+a_{1}(n)\right) n+2\left(a_{0}(n)+a_{1}(n)\right)} \sum_{k=0}^{n} p_{n, k}(x)\left(\sum_{r=0}^{N} w_{r} p_{n, k}^{M, 1}\left(x_{r}\right) f\left(x_{r}\right)\right) .
$$

It is worthy to point out that, in our numerical results, we will use a simple and reliable integration rule which is well-known as composite Simpson's rule. To testify the integration rules (7.1), some example are provided. If we denote

then

$$
I_{N}^{n, k}(f):=\int_{0}^{1} p_{n, k}^{M, 1}(x) f(x) \mathrm{d} x
$$

$$
E_{N}(f)=\left|I_{N}^{n, k}(f)-\sum_{r=0}^{N} w_{r} p_{n, k}^{M, 1}\left(x_{r}\right) f\left(x_{r}\right)\right| .
$$

In Table 1, the absolute errors $E_{N}(f)$ of some functions for some values of $N$ with $n=10$ and $k=5$ are reported.

TABLE 1. Comparison of the absolute errors $E_{N}(f)$ of some given functions for some values of $N$ with $n=10$ and $k=5$.

\begin{tabular}{llll}
\hline \hline & & \\
$N$ & $E_{N}\left(x^{5}\right)$ & $E_{N}(\cos (x))$ & $E_{N}\left(x^{2} \sin \left(x^{2}\right)\right)$ \\
\hline & & & \\
10 & $1.46998 e-06$ & $1.36821 e-06$ & $1.13374 e-06$ \\
20 & $2.65594 e-08$ & $2.18601 e-08$ & $1.93467 e-08$ \\
30 & $2.39334 e-09$ & $1.93040 e-09$ & $1.72519 e-09$ \\
40 & $4.29848 e-10$ & $3.47300 e-10$ & $3.08712 e-10$ \\
50 & $1.13155 e-10$ & $9.40214 e-11$ & $8.11280 e-11$ \\
60 & $3.79818 e-11$ & $3.41313 e-11$ & $2.72051 e-11$ \\
70 & $1.50831 e-11$ & $1.59270 e-11$ & $1.07964 e-11$ \\
80 & $6.77523 e-12$ & $9.33101 e-12$ & $4.84690 e-12$ \\
90 & $3.34406 e-12$ & $6.60918 e-12$ & $2.39075 e-12$ \\
100 & $1.77794 e-12$ & $5.36758 e-12$ & $1.26999 e-12$ \\
\hline
\end{tabular}

One can easily see that the obtained errors for $N \geq 80$ are reliable. So, in the rest of this paper, we will use composite Simpson's rule with $N=100$.

Now and for the third step, the Durrmeyer and Modified-Bernstein-Durrmeyer operators will be used to approximate some smooth and non-smooth functions. For the first example, consider a smooth function $\cos (x)$ in 

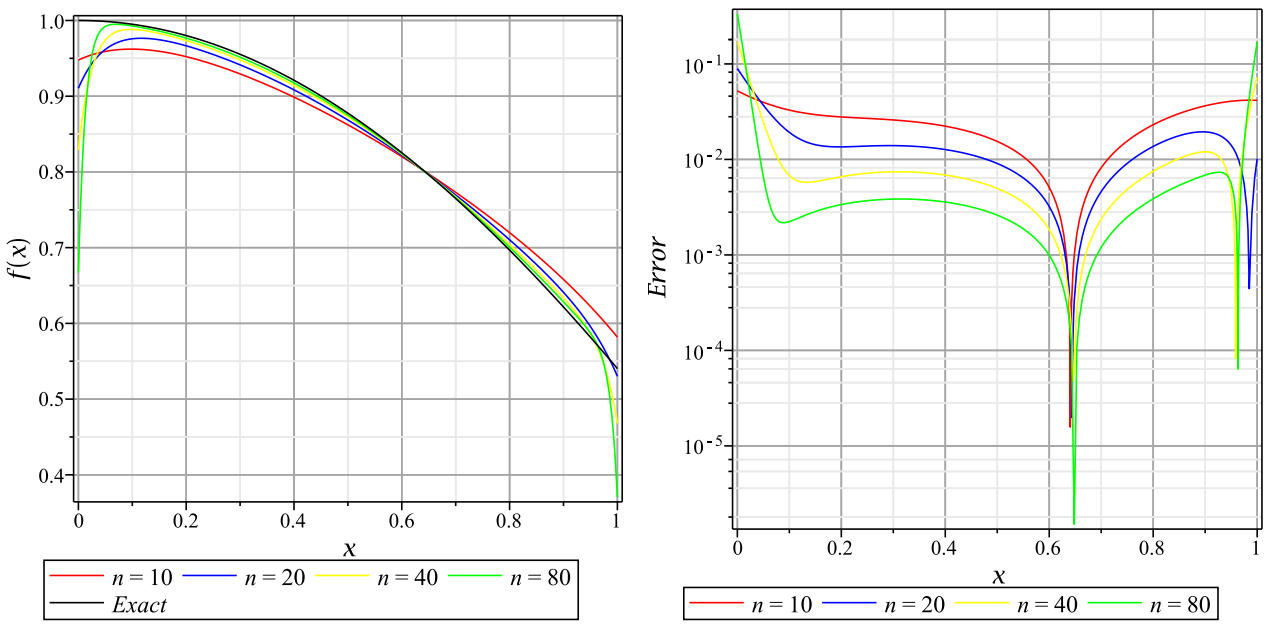

FiguRE 2. Comparison of the behavior of the approximation results and the absolute errors of the Durrmeyer operator $D_{n}(f ; x)$ for some values of $n$.
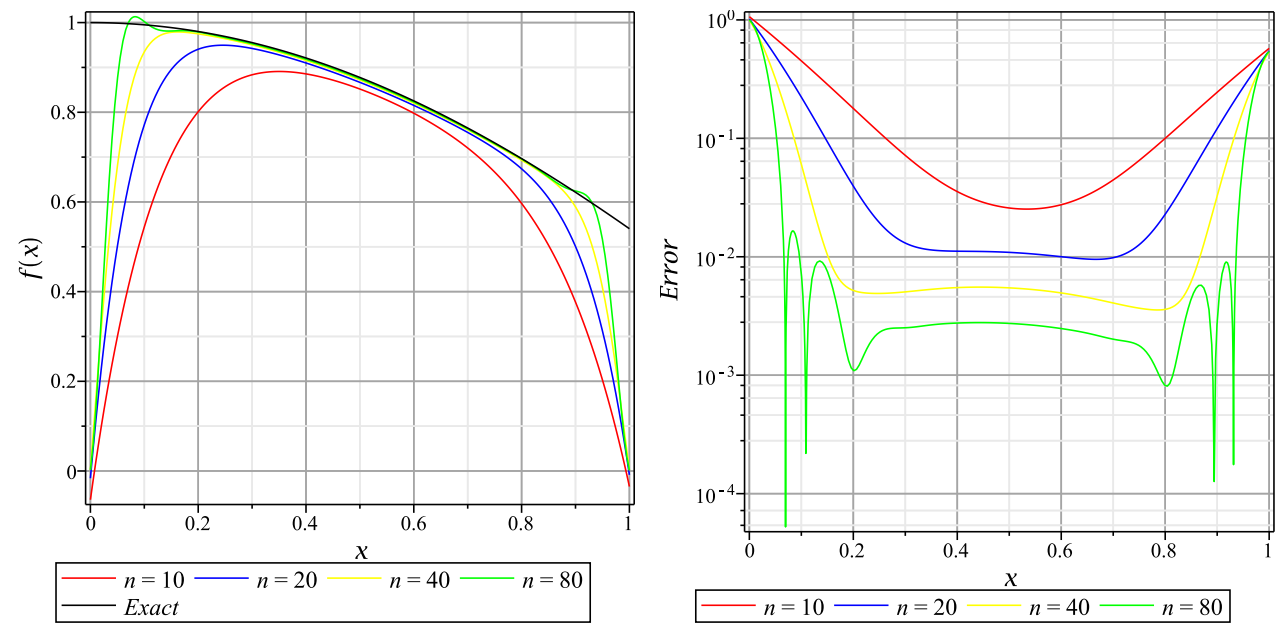

FIGURE 3. Comparison of the behavior of the approximation results and the absolute errors of the Modified-Bernstein-Durrmeyer operator $D_{n}^{M, 1}(f ; x)$ for some values of $n$.

$[0,1]$. The approximation results for both operators with some values of $n$ are shown in Figs. 2 and 3 It can be observed from these figures that, however, obtained errors decay as long as $n$ is increased. Moreover, the absolute errors reach their maximum values the end points $x=0$ and $x=1$. 
For the second example, the following non-smooth concave functions [23 are considered.

$$
f(x)= \begin{cases}8 x, & 0 \leq x \leq 0.2 \\ \frac{4}{3}(1+x), & 0.2 \leq x \leq 0.5 \\ \frac{4}{3}(2-x), & 0.5 \leq x \leq 0.8 \\ 8(1-x), & 0.8 \leq x \leq 1\end{cases}
$$

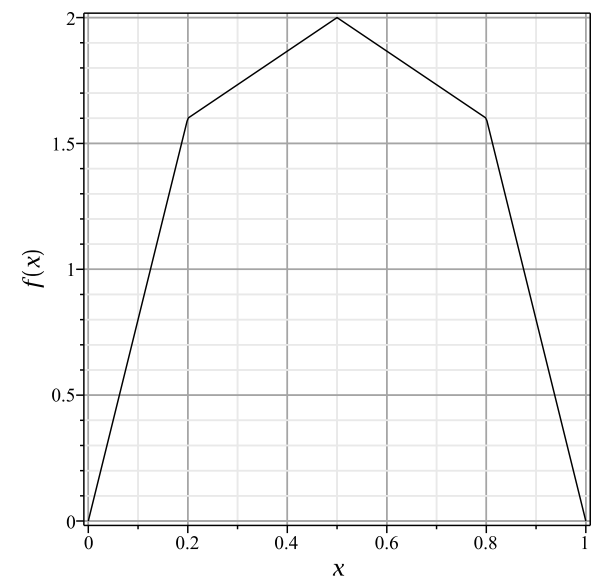

Figure 4. Plot of function (7.3).

It can be easily checked that, however, $f \in C[0,1]$ but it is not differentiable at some interior points $x=0.2,0.5,0.8$. The approximation results together with the absolute errors for both operators $D_{n}(f ; x)$ and $D_{n}^{M, 1}(f ; x)$ for various values of $n$ are depicted in Figs. 5 and 6, respectively. The results clearly show that the absolute errors of the
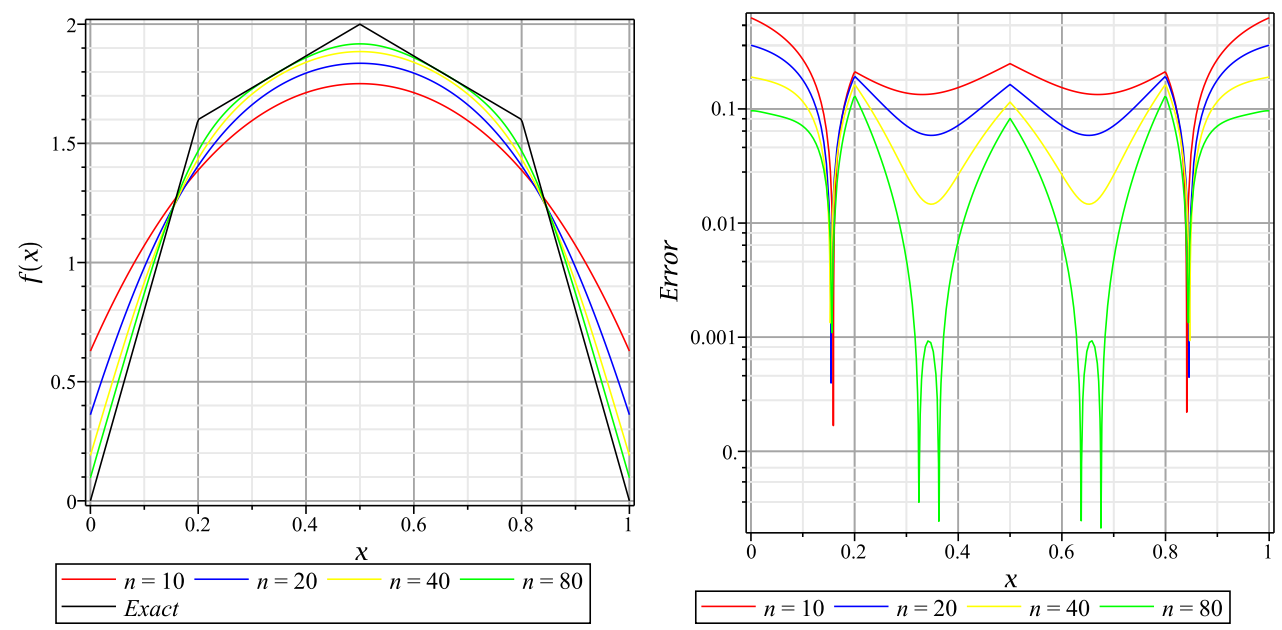

Figure 5. Comparison of the behavior of the approximation results and the absolute errors of the Durrmeyer operator $D_{n}(f ; x)$ for some values of $n$.

Modified-Bernstein-Durrmeyer operator $D_{n}^{M, 1}(f ; x)$ are better than those for Durrmeyer operator $D_{n}(f ; x)$. For the third example, the following non-smooth function is studied: 

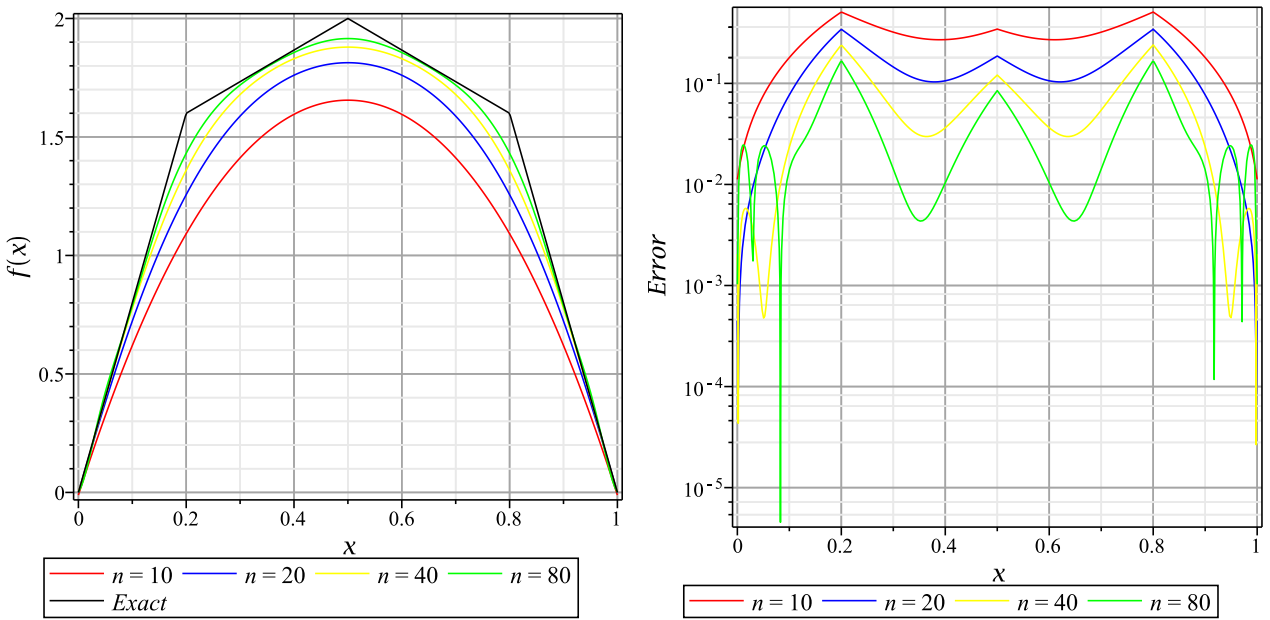

FiguRE 6. Comparison of the behavior of the approximation results and the absolute errors of the Modified-Bernstein-Durrmeyer operator $D_{n}^{M, 1}(f ; x)$ for some values of $n$.

$$
f(x)= \begin{cases}-25 x+5, & 0 \leq x \leq 0.2 \\ 40(x-0.8)(0.2-x), & 0.2 \leq x \leq 0.8 \\ -25(1-x)+5, & 0.8 \leq x \leq 1\end{cases}
$$

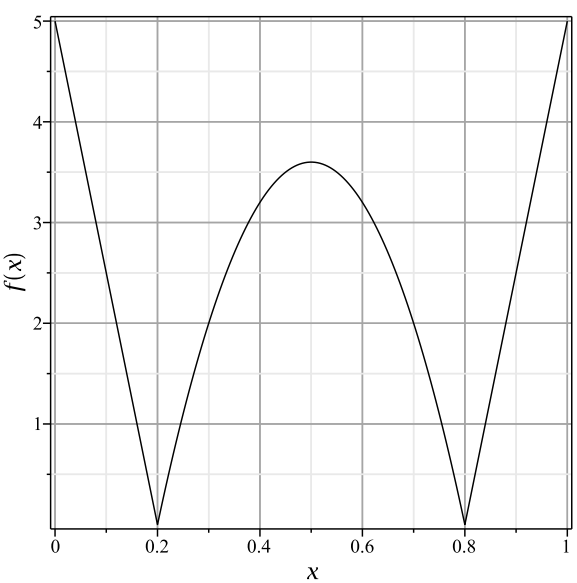

Figure 7. Plot of function (7.4).

We also note that for this example we have $f \in C[0,1]$ and $f$ is not differentiable at $x=0.2,0.8$.

Comparison of the approximation results together with the absolute errors for both operators $D_{n}(f ; x)$ and $D_{n}^{M, 1}(f ; x)$ for various values of $n$ are plotted in Figs. 8 and 9 respectively. The obtained results show that, in this example, the absolute errors of the Durrmeyer operator $D_{n}(f ; x)$ are better than those for the ModifiedBernstein-Durrmeyer operator $D_{n}^{M, 1}(f ; x)$.

In conclusion, the results obtained from these examples suggest that for functions vanish at the end points $x=0$ and $x=1$, the Modified-Bernstein-Durrmeyer operator $D_{n}^{M, 1}(f ; x)$ yields better results while for the other functions the Durrmeyer operator $D_{n}(f ; x)$ works good.

Here and for the last step of this section, we are going to verify the Point-wise Order of Convergence (POC) of the newly operator $D_{n}^{M, 1}(f ; x)$. To analyze the POC proved in Theorems 3 and 6 , we first denote

$$
\operatorname{POC}(n)=\left|\frac{\log _{10}\left(\frac{E_{n+1}(f)}{E_{n}(f)}\right)}{\log _{10}\left(\frac{n}{n+1}\right)}\right|, E_{n}(f)=\left|p_{n}^{M, 1}(f ; 0.5)-f(0.5)\right| .
$$

For the first example consider $f(x)=\cos (x)$. We know that $f \in C^{\infty}[0,1]$. The POC of the operators $D_{n}(f ; x)$ and $D_{n}^{M, 1}(f ; x)$ with respect to the function $f(x)=\cos (x)$ for various values of $n$ are reported in columns two and three of Table 2 The results of the second and third columns of this table show that the POC of these operators (with respect to the aforementioned function) is equal to 1 which verify the theoretical result presented in Voronovskaya theorem 6 .

For the second example consider function (7.3). We also note that $f \in C[0,1]$ and also in not differentiable on $[0,1]$. The POC of the operators $D_{n}(f ; x)$ and $D_{n}^{M, 1}(f ; x)$ with respect to this function for various values of $n$ are 

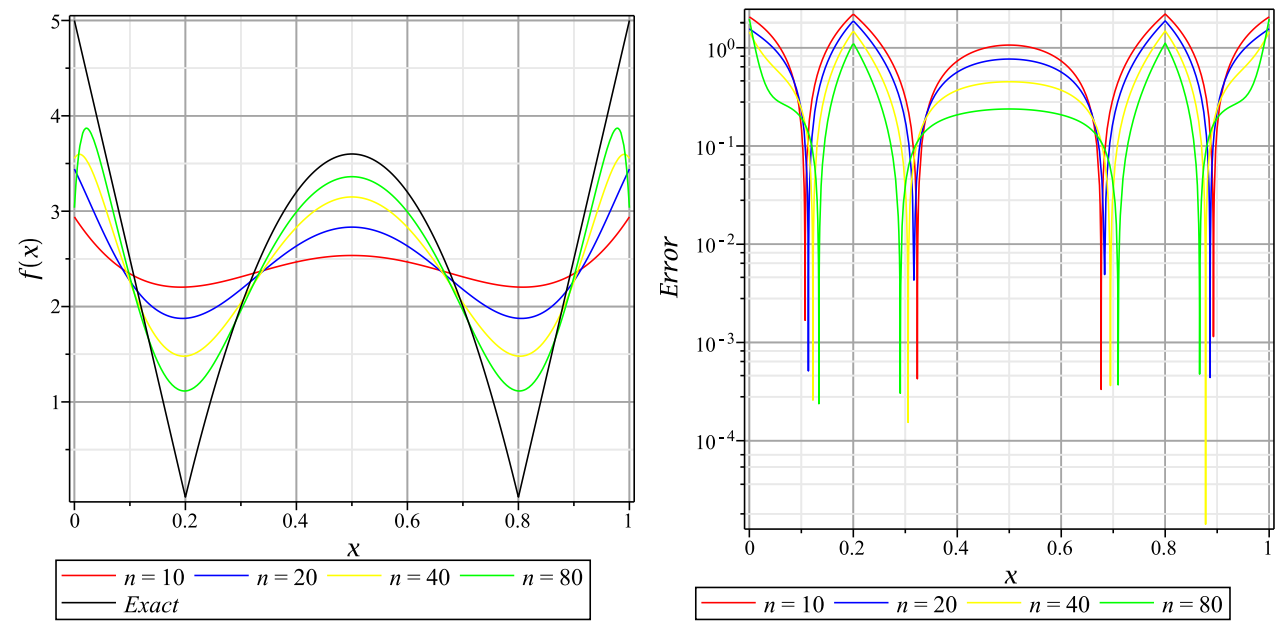

FiguRE 8. Comparison of the behavior of the approximation results and the absolute errors of the Durrmeyer operator $D_{n}(f ; x)$ for some values of $n$.
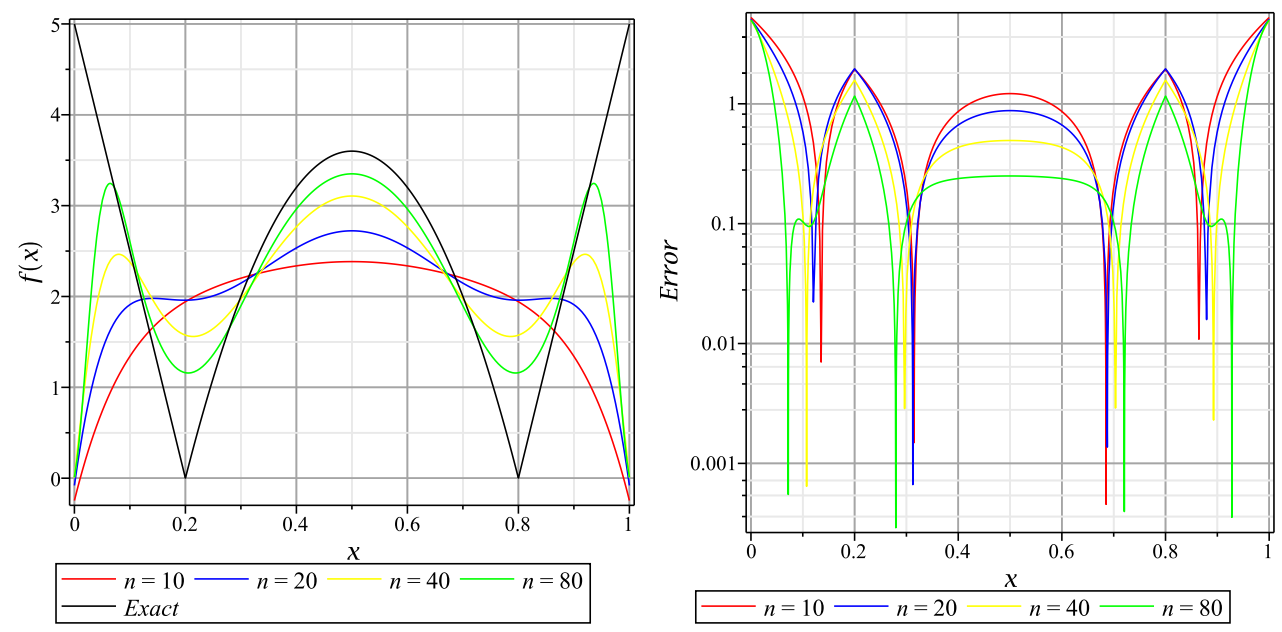

Figure 9. Comparison of the behavior of the approximation results and the absolute errors of the Modified-Bernstein-Durrmeyer operator $D_{n}^{M, 1}(f ; x)$ for some values of $n$.

reported in columns four and five of Table 2. The obtained results which tend to 0.5 are also verified the result of Theorem (3).

\section{Conclusion}

In this paper, we studied approximation properties of a new integral variant of Bernstein operators. First, we obtained error estimates in terms of the ordinary modulus of continuity and then we generalised the result in terms of the Ditzian-Totik modulus of continuity. Subsequently, for a larger class of functions(the class of functions of bounded variation) an upper bound for rate of approximation was proved. A number of suitable numerical examples were studied for the convergence properties. The researchers can use the technique of this paper to investigate approximation properties of many other sequences of linear positive operators. Further, researchers may find it interesting to consider the operators for $L p$ as well as weighted approximation.

Acknowledgements. The authors express their gratitude to the learned reviewers for their valuable comments which were very helpful to improve the manuscript.

Funding: This study was funded by MHRD/UGC - Empowered Committees Basic Science Research Programme (No.F.30-371/2017(BSR). 
TABLE 2. The POC of the operators $D_{n}(f ; x)$ and $D_{n}^{M, 1}(f ; x)$ with respect to the function $f(x)=$ $\cos (x)$ for various values of $n$.

\begin{tabular}{lccccc}
\hline \hline & \multicolumn{2}{c}{$f(x)=\cos (x)$} & & \multicolumn{2}{c}{ function $\sqrt{7.3 p}$} \\
\cline { 2 - 3 }$n$ & $D_{n}(f ; x)$ & $D_{n}^{M, 1}(f ; x)$ & & $D_{n}(f ; x)$ & $D_{n}^{M, 1}(f ; x)$ \\
\hline & & & & \\
10 & 1.36079 & 0.70836 & 0.98624 & 0.6287 \\
20 & 0.99099 & 0.82667 & 0.73765 & 0.55715 \\
30 & 0.99328 & 0.87796 & 0.59684 & 0.50128 \\
40 & 0.99532 & 0.90584 & 0.53986 & 0.48085 \\
50 & 0.99645 & 0.92334 & 0.51827 & 0.47602 \\
60 & 0.99717 & 0.93536 & 0.50999 & 0.47655 \\
70 & 0.99765 & 0.94412 & 0.50663 & 0.47854 \\
80 & 0.99821 & 0.95078 & 0.50512 & 0.48068 \\
90 & 0.99826 & 0.95603 & 0.50428 & 0.4826 \\
100 & 0.99846 & 0.96026 & 0.50376 & 0.48423 \\
\hline
\end{tabular}

\section{Compliance with Ethical Standards}

Conflict of interest. The authors declare that they have no conflict of interest.

This article does not contain any studies with human participants performed by any of the authors.

\section{REFERENCES}

[1] Abel U, Ivan M (2011) New representation of the remainder in the Bernstein approximation. J. Math. Anal. Appl. 381, 952-956.

[2] Acu AM, Gupta V, Tachev G (2019) Better numerical approximation by durrmeyer type operators, Results Math. 74:90.

[3] Adell JA, Bustamante J, Quesada JM (2015) Sharp upper and lower bounds for the moments of Bernstein polynomials. Appl. Math. Comput. 265, 723-732.

[4] Adell JA, de la Cal J (1995) Bernstein-Durrmeyer operators, Computers Math. Applic. Vol. 30, No. 3-6, pp. 1-14.

[5] Aramă O, Ripianu D (1961) Une proprit des polynomes de Bernstein. Mathematica (Cluj) 3(26), 518 (in French).

[6] Agrawal PN, Gupta V (1990) On the iterative combination of Phillips operators, Bull. Inst. Math. Acad. Sinica, 18, 4, 361-368.

[7] Bernstein SN (1912) Démonstration du théorème de Weierstrass fondée, Comm. Kharkov Math. Soc. 13, 1-2.

[8] Bustamante J (2017) Bernstein Operators and Their Properties, Springer International Publishing.

[9] Bustamante J, García-Muñoz MA, Quesada JM (2014) Bernstein polynomial and discontinuous functions. J. Math. Anal. Appl. 411, 829-837.

[10] Butzer PL (1953) Linear combinations of Bernstein polynomials, Canad. J. Math., 5, 559-567.

[11] Ditzian Z, Totik V (1987) Moduli of Smoothness, Springer, New York.

[12] Ditzian Z, Ivanov K (1989) Bernstein Type Operators and their derivatives, J. Approx. Theory, 56, 72-89.

[13] Draganov BS (2014) On Simultaneous approximation by iterated Boolean sums of Bernstein operators, Results. Math. 66, 21-41.

[14] Durrmeyer JL (1967) Une formule d'inversion de la transformée de Laplace: applications à la théorie des moments, Thése de 3e cycle, Paris.

[15] Derriennic MM (1978) Sur lapproximation des fonctions d'une ou plusieurs variables par des polynômes de Bernstein modifiés et application au problème des moments, Thèse de 3e cycle, Université de Rennes.

[16] Derriennic MM (1981) Sur lapproximation de fonctions intégrables sur [0,1] par des polynômes de Bernstein modifiés, J. Approx. Theory, 31, 325-343.

[17] Gonska HH, Zhou XL (1991) A global inverse theorem on simultaneous approximation by Bernstein-Durrmeyer operators, J. Approx. Theory 67, no. 3, 284-302.

[18] Gupta V, Agarwal RP (2014) Convergence Estimates in Approximation Theory. Springer, New York.

[19] Gupta V, Ispir N (2004) On simultaneous approximation for some modified Bernstein type operators. Int. J. Math. Math. Sci., $71,3951-3958$

[20] Gupta V, Maheshwari P (2003) Bézier variant of a new Durrmeyer type operators, Rivista di Matematica della Università di Parma 7(2), 9-21.

[21] Heilmann M (1992) Erhöhung der Konvergenzgeschwindigkeit bei der Approximation von Funktionen mit Hilfe von Linearkombinationen spezieller positiver linearer Operatoren. Habilitationsschrift, Universitt Dortmund.

[22] Kantorovich LV (1930) Sur certain développements suivant les polynômes de la forme de S. Bernstein, I, II, C. R. Acad. URSS, $563-568,595-600$.

[23] Khosravian-Arab H, Dehghan M, Eslahchi MR (2018) A new approach to improve the order of approximation of the Bernstein operators: Theory and applications, Numer. Algorithms 77, no. 1, 111-150.

[24] Korovkin PP (2017) Linear Operators and Approximation Theorey, First revised edition, Hindustan Pub. Cor.

[25] Lorentz GG (1953) Bernstein Polynomials, Univ. of Toronto Press.

[26] Lupaş A (1972) Die Folge der Betaoperatoren, Dissertation, Universität Stuttgart. 
[27] Micchelli CA (1973), The saturation class and iterates of the Bernstein polynomials, J. Approx. Theory, 8, 1-18.

[28] Özarslan MA , Aktuğlu H (2013) Local approximation for certain King type operators, Filomat 27(1), 173-181.

[29] Popoviciu T (1935) Sur lapproximation des fonctions convexes dordre superieur. Mathematica (Cluj) 10, 49-54(in French).

[30] Rivlin JT (2003) An Introduction to the Approximation of Functions, Courier Corporation.

[31] Stancu DD, Coman Gh, Agratini O (2001) Trîmbiţaş, R., Analiză numeric ă şi teoria aproximării, I, Presa Universitară Clujeană, Cluj-Napoca,(Romanian).

[32] Stoer J, Bulirsch R (2013) Introduction to numerical analysis, Vol. 12. Springer Science \& Business Media. 